\title{
Lyophilized powder of mesenchymal stem cell supernatant attenuates acute lung injury through the IL-6-p-STAT3-p63-JAG2 pathway
}

\section{Wenjun Peng}

Zhongshan Hospital Fudan University

\section{Meijia Chang}

Zhongshan Hospital Fudan University

\section{Yuanyuan Wu}

Zhongshan Hospital Fudan University

Wensi Zhu

Zhongshan Hospital Fudan University

\section{Lin Tong}

Zhongshan Hospital Fudan University

\section{Ge Zhang}

Zhongshan Hospital Fudan University

Qin Wang

Zhongshan Hospital Fudan University

Jie Liu

Zhongshan Hospital Fudan University

\section{Xiaoping Zhu}

Tongji University Affilliated East Hospital: Shanghai East Hospital

\section{Tingting Cheng}

Tongji University Affilliated East Hospital: Shanghai East Hospital

\section{Yijia Li}

Yangtze Delta Region Institute of Tsinghua University

\section{Xi Chen}

Yunnan Province Stem cell Bank

\section{Dong Weng}

Tongji University Affiliated Shanghai Pulmonary Hospital

\section{Sanhong Liu}

Shanghai University of Traditional Chinese Medicine

\section{Hongwei Zhang}

Shanghai University of Traditional Chinese Medicine

Yao Su 
Yangtze Delta Region Institute of Tsinghua University

Jian Zhou ( $\square$ zhou.jian@fudan.edu.cn )

Zhongshan Hospital Fudan University https://orcid.org/0000-0003-4736-9457

\section{Huayin Li}

Zhongshan Hospital Fudan University

\section{Yuanlin Song}

Zhongshan Hospital Fudan University

\section{Research}

Keywords: Acute lung injury, Bleomycin, Mesenchymal stem cells, Secretome, Lyophilized powder, p63, Lipidomics

Posted Date: October 2nd, 2020

DOI: https://doi.org/10.21203/rs.3.rs-85300/v1

License: (c) (i) This work is licensed under a Creative Commons Attribution 4.0 International License. Read Full License

Version of Record: A version of this preprint was published at Stem Cell Research \& Therapy on March 29th, 2021. See the published version at https://doi.org/10.1186/s13287-021-02276-y. 


\section{Abstract}

Background: Mesenchymal stem cells (MSCs) may contribute to repair in severe diseases including acute lung injury (ALI). However, applications with MSCs have been restricted due to safety considerations and limitations in terms of large-scale production and industrial delivery. Alternatively, the MSC secretome has been considered promising for use in therapeutic approaches and has been advanced to pre-clinical and clinical trials. Furthermore, the MSC secretome can be freeze-dried into a stable and ready-to-use supernatant lyophilized powder (SLP) form.

Methods: Intratracheal bleomycin was used to induce ALI in mice, and intratracheal MSC SLP was delivered as a treatment. Histopathological assessment was achieved by hematoxylin and eosin, immunohistochemistry, and immunofluorescence staining. Apoptosis, inflammatory infiltration, immunological cell counts, cytokine levels, and mRNA- and protein-expression levels of relevant targets were measured by performing terminal deoxynucleotidyl transferase dUTP nick-end labeling assays, determining total cell and protein levels in bronchoalveolar lavage fluids, flow cytometry, multiple cytokine-detection techniques, and reverse transcriptase-quantitative polymerase chain reaction and western blot analysis, respectively. Plasma lipid profiles were detected by performing lipidomics analysis.

Results: Here, we found that intratracheal MSC SLP considerably promoted cell survival, inhibited epithelial cell apoptosis, attenuated inflammatory cell recruitment, and reversed immunological imbalances induced by bleomycin. MSC SLP inhibited signaling through the interleukin 6phosphorylated signal transducer and activator of transcription pathway to activate tumor protein 63jagged 2 signaling in basal cells, suppress $T$ helper 17 cell differentiation, promote $p 63^{+}$cell proliferation and lung damage repair, and relieve inflammatory responses. Furthermore, MSC SLP significantly reversed changes in plasma lipid profiles caused by bleomycin.

Conclusions: MSC SLP ameliorated ALI by activating p63 and promoting $p 63^{+}$cell proliferation and the repair of damaged epithelial cells. The findings of this study also shed insight into ALI pathogenesis and imply that MSC SLP shows considerable therapeutic promise for treating ALI and acute respiratory distress syndrome. Our findings also suggest that phosphatidylserine has potential for serving as a pharmaceutical or dietary supplement for alleviating ALI.

\section{Background}

Acute lung injury (ALI) and its most severest manifestation, acute respiratory distress syndrome (ARDS), are characterized by clinical hypoxemia combined with bilateral pulmonary infiltrates and edema, which results in high mortality (1). Although lung-protective ventilation can improve airway pressure to a certain extent, no specific treatment has been demonstrated to be satisfactory, and efficient pharmaceuticals are still urgently needed. Bleomycin (BLM) is a chemotherapeutic drug used against various human malignancies that is also widely applied in murine models to induce ALI at the inflammatory phase (within 1 week) or pulmonary fibrosis at the fibrotic phase (during the second week) $(2,3)$. 
Mesenchymal stem cells (MSCs) are multipotent cells, which have attracted much attention due to their potential in regenerating defective tissues and their immunomodulatory capabilities in inflammatory disorders (4). MSCs were initially found in the bone marrow and then isolated from most postnatal organs (5) such as adipose tissue (6), the lungs (7), and tangential blood (8). Fetal tissues including the placenta and umbilical cord are also available sources of MSCs (9). Over the past decades, MSCs and the MSC secretome have shown great promise for treating a great assortment of diseases, especially severe disorders without effective pharmacotherapies $(10,11)$. Previously, MSC therapy showed good efficacy against ALI, based on the potentials of MSC colonization and differentiation, and the abilities of MSCs to secrete soluble bioactive molecules $(12,13)$. However, MSCs are inconvenient to produce and store, and the risks of iatrogenic tumor formation and pulmonary embolism remain as concerns for MSC therapy (14). Because MSC engraftment is transient, much of the current research related to MSC therapy has focused on the paracrine-signaling capacity and the MSC secretome. In this study, the secretome of placenta-derived MSCs was collected and purified from culture supernatants and then freeze-dried into an MSC supernatant lyophilized powder (MSC SLP) form. The specific procedure for preparing MSC SLP is presented in Supplementary Fig. 1.

Interleukin-6 (IL-6), which is primarily secreted by macrophages and type-2 pneumocytes in the lungs, has been commonly identified as a vital pro-inflammatory cytokine involved in various inflammatory disorders of the lungs (15-18). IL-6 has also been implicated in the pathogenesis of BLM-induced inflammatory injury and subsequent fibrosis $(19,20)$. At the inflammatory stage, BLM-induced IL- 6 is produced predominantly by type- 2 pneumocytes and functions as a pro-inflammatory and anti-fibrosis factor (20), and IL-6 deficiency attenuated inflammatory cell recruitment (19). At the fibrotic stage, BLMinduced IL-6 is produced mainly by macrophages and fibroblasts and has pro-fibrotic activity (20).

Basal cells are multipotent tissue-specific epithelial progenitors that express tumor protein 63 (p63, also known as Trp63), cytokeratin 5 (Krt5), and cytokeratin 14 (Krt14), which function as stem cells that proliferate rapidly following epithelial injury and help regenerate damaged epithelium in both mouse trachea and human airways $(21,22)$. Normally, basal cells are relatively quiescent, as they can rapidly self-renew and differentiate in response to stimulation (21). As a member of p53 family, p63 is highly expressed in basal cells of various epithelial tissues and confers stem cell properties $(23,24)$. Similarly, p63 has been shown to be a vital mediator of the normal development, maintenance, and homeostasis of the epithelium $(23,25)$. In addition, p63 has been widely documented in various epithelial tumors, including squamous cell carcinomas of the lungs, prostate, and bladder $(26,27)$.

Lipids are composed of diverse classes of biomolecules and are often overlooked, due to their relative inertness. However, lipids are indispensable in nearly all aspects of cellular functions, such as the maintenance of energy balance, membrane homeostasis, and cellular signaling pathways. For instance, phosphatidylcholine (PC) and phosphatidylethanolamine (PE), well known as major components of cell membranes, are associated with cell proliferation, differentiation, and apoptosis $(28,29)$. In the lungs, lipids play significant roles in normal development and in various diseases. It was previously shown that dipalmitoylphosphatidylcholine, a primary component of pulmonary surfactant material, can reduce 
alveolar surface tension and prevent the early onset of ALI, due to its specific biophysical properties (30). Lipids have been closely associated with BLM-induced ALI and pulmonary fibrosis $(31,32)$.

Understanding global lipid profiles aids in elucidating the mechanisms underlying ALI and discovering potential therapeutic targets.

In this study, we established a mouse model of BLM-induced ALI and compared distinct characteristics before and after MSC SLP treatment, in order to evaluate the mechanism whereby MSC SLP can attenuate ALI.

\section{Materials And Methods}

\section{MSC SLP preparation}

Placenta-derived MSCs were isolated from human placental chorion samples from newborns. Chorion tissue blocks were cultured in a-MEM with 7\% human platelet lysate (UltraGRO-Advanced, AventaCell Biomedical, Atlanta, GA, USA). Approximately 7-10 days later, the primary cells could be passaged to passage 1 (P1). When the $\mathrm{P} 1$ cell density reached $80 \%$, the culture could be passaged to $\mathrm{P} 2$. In the same manner, the cells were passed from $\mathrm{P} 2$ to $\mathrm{P} 3$. When the $\mathrm{P} 3$ cell density reached approximately $70 \%$, placenta-derived MSCs were washed three times with phosphate-buffered saline (PBS) and cultured in human platelet lysate-free a-MEM medium for $24 \mathrm{~h}$. Then, supernatants from placenta-derived MSCs were collected and processed into lyophilized powder. The procedure used is shown in detail in Supplementary Fig. 1. MSC SLP was sealed and stored at $-20^{\circ} \mathrm{C}$ for later use.

\section{Animals}

Eight-week-old male C57BL/ 6 mice were routinely bred in the animal facility of Zhongshan Hospital at Fudan University (Shanghai, China). The mice were housed at $20-25^{\circ} \mathrm{C}$ with a relative humidity of $50-$ $70 \%$ and were provided free access to water and food. The experimental procedures were approved by the Animal Care and Use Committee of Zhongshan Hospital at Fudan University. Intratracheal BLM $(2.5 \mathrm{mg} / \mathrm{kg}$ ) was instilled in mice to induce ALI, and mice in the control group received an equal volume of PBS. MSC SLP (50 mg/kg) was dissolved in PBS and delivered intratracheally $1 \mathrm{~h}$ after BLM instillation. The mice were sacrificed to collect peripheral blood, bronchoalveolar lavage fluids (BALFs), and lung tissues on day 7. To evaluate the role of IL-6 in ALI, recombinant human (rh) IL-6 (PeproTech, \#200-06, NJ, USA) was administrated intratracheally at a dose of $1,000 \mathrm{ng}$ per mouse. MSC SLP $(50 \mathrm{mg} / \mathrm{kg})$, dissolved in PBS, was also administered intratracheally $1 \mathrm{~h}$ after $\mathrm{rh}$ IL-6 instillation, and the mice were sacrificed on day 2.

\section{Hematoxylin and eosin (H\&E) staining}

The right upper lobes of the lung samples were harvested, fixed in $4 \%$ paraformaldehyde overnight, and embedded in paraffin. Lung sections were stained with $\mathrm{H} \& \mathrm{E}$, and pathological-damage scores were calculated to assess lung injury as follows: no injury $=0$; injury in less than $25 \%$ of the field $=1$; injury in 
$25-50 \%$ of the field $=2$; injury in $50-75 \%$ of the field $=3$; injury in more than $75 \%$ of the field $=4$. Ten fields were randomly selected and assessed by investigators blinded to the grouping.

\section{Total protein concentrations and inflammatory cell counts in BALFs}

BALFs were collected by cannulating the trachea and centrifuging at $1,000 \mathrm{rpm}$ for $5 \mathrm{~min}$ at $4{ }^{\circ} \mathrm{C}$. Each supernatant was stored at $-80^{\circ} \mathrm{C}$, and each cell pellet was resuspended and stained with Wright-Giemsa staining solution (Thermo Fisher Scientific, Waltham, MA, USA). Cell-free supernatants were used to measure total protein levels with the Bicinchoninic Acid (BCA) Protein Assay Kit (7780, Cell Signaling Technology [CST], Boston, MA, USA).

\section{Myeloperoxidase (MPO) activity determinations}

The left lungs of mice were weighted and ground into tissue homogenates. MPO activities in lung homogenates were determined using the MPO Assay Kit (ab105136, Abcam, Cambridge, MA, USA), following the manufacturer's instructions.

\section{Flow cytometry}

Harvested lungs were minced mechanically, digested with an enzyme mix (buffer $S$, enzyme $D$, and enzyme A from Miltenyi Biotec, Bergisch Gladbach, Germany), washed, and re-suspended in PBS. Heparin, antibodies, and red blood cell lysis buffer were sequentially added to whole blood samples. Cells were stained with a fluorescein isothiocyanate (FITC)-conjugated antibody against cluster of differentiation (CD) protein 4, an allophycocyanin (APC)-conjugated antibody against CD25, and a phycoerythrin-conjugated antibody against forkhead box P3 (Foxp3) to detect regulatory T (Treg) cells. In addition, staining with CD3-FITC and CD8-APC antibodies was performed to detect CD $4^{+} \mathrm{T}$ cells $\left(\mathrm{CD} 3^{+} \mathrm{CD} 8^{-} \mathrm{T}\right.$ cells), and intracellular staining against IL-17A was performed to detect Thelper 17 (Th17) cells. The antibodies were purchased from eBioscience (San Diego, CA, USA), and staining was performed according to the manufacturer's instructions.

\section{Apoptosis detection}

To identify apoptotic cells, terminal deoxynucleotidyl transferase dUTP nick-end labeling (TUNEL) assays were performed using an In Situ Cell Death Detection Kit (Roche, Indianapolis, IN, USA). All procedures were performed in accordance with the manufacturer's instructions.

\section{Immunofluorescence staining}

Lung sections were incubated in $5 \%$ goat serum to block nonspecific binding, followed by incubation with primary antibodies overnight at $4^{\circ} \mathrm{C}$. Primary antibodies against p63 (ab53039, Abcam), alpha smooth muscle actin (a-SMA; Servicebio, Wuhan, China), and Ki-67 (ab15580, Abcam) were used. After washing the sections three times in PBS (10 min each wash), they were incubated with an appropriate secondary antibody for $2 \mathrm{~h}$ in the dark at $21^{\circ} \mathrm{C}$. The secondary antibodies used included an Alexa Fluor 488conjugated goat anti-rabbit IgG (4412, CST), a cyanine 3-conjugated goat anti-mouse IgG (GB21301, Servicebio) and a horseradish peroxidase (HRP)-conjugated goat anti-rabbit IgG (GB23303, Servicebio). 
Cover slips were applied for mounting. The sections were imaged under a fluorescence microscope (Eclipse C1, Nikon, Tokyo, Japan), using a $\times 40$ objective.

\section{Cytokine measurements}

To determine the concentrations of granulocyte-macrophage colony-stimulating factor (GM-CSF), interferon (IFN)- $\gamma$, IL-10, IL-13, IL-17A, IL-17C, IL-17F, IL-1b, IL-4, IL-6, IL-23, and tumor necrosis factor (TNF)- $a$ in plasma samples, we performed Meso Scale Discovery ultrasensitive multifactor electrochemiluminescence assays (Meso Scale Discovery, Rockville, MD, USA), according to the manufacturer's instructions. Then, plasma IL- 6 concentrations and BALF IL- 6 and IL-1 $\beta$ concentrations were measured using corresponding enzyme-linked immunosorbent assay (ELISA) kits from eBioscience (San Diego, CA, USA).

\section{Real-time reverse transcriptase-quantitative polymerase chain reaction (RT-qPCR) analysis}

Total RNA was extracted from the lungs of mice using the TRIzol Reagent (Thermo Fisher Scientific) and reverse-transcribed into complementary DNA using a reverse transcriptase kit (Toyobo, Osaka, Japan). mRNA-expression levels were quantified using SYBR Premix EX Taq ${ }^{\text {TM }}$ (TaKaRa Bio, Osaka, Japan) with $\beta-$ actin expression serving as an internal control. Primers with the following sequences were used for the qPCR step: Trp63: forward: 5'-TTG TGA AAC GAT GCC CTA AC-3'; reverse: 5'-CTC TGC CTT CCC GTG ATA3'; JAG2: forward: 5'-CCT GTG TGG TTA TCT GCG TAT-3'; reverse: 5'-GCT CTC ATC CCG TGG TAG-3'; $\beta$ actin: forward: 5'-CCT CTA TGC CAA CAC AGT-3'; reverse: 5'-AGC CAC CAA TCC ACA CAG-3'.

\section{Western blot analysis}

Lung tissues were lysed and homogenized in radioimmunoprecipitation assay buffer with phosphatase inhibitors and protease inhibitors (Beyotime Biotechnology). Each homogenate was centrifuged at $12,000 \mathrm{rpm}$ for $15 \mathrm{~min}$ at $4^{\circ} \mathrm{C}$ to extract proteins, and the concentrations was determined by performing BCA protein assays $(7780, C S T)$. Equal amounts of protein from each sample were resolved by sodium dodecyl sulfate (SDS)-polyacrylamide gel electrophoresis and then transferred to polyvinylidene fluoride membranes. After blocking at room temperature for $1 \mathrm{~h}$, the membranes were incubated overnight at $4{ }^{\circ} \mathrm{C}$ with antibodies against p63 (ab53039, Abcam), phosphorylated signal transducer and activator of transcription (p-STAT3; 9145, CST), STAT3 (9139, CST), jagged 2 (JAG2; NBP1-58284, Novus Biologicals, Centennial, CO, USA), and $\beta$-actin $(4970$, CST). After washed three times with Tris-buffered saline with Tween-20, the membranes were incubated with an appropriate HRP-conjugated secondary antibody at room temperature for $1 \mathrm{~h}$. Protein bands were exposed with enhanced electro-chemiluminescence reagents (Beyotime Biotechnology), and the bands were analyzed with an Imaging System (Bio-Rad, CA, USA).

\section{Immunohistochemistry (IHC)}

The lung sections were incubated at $56^{\circ} \mathrm{C}$ for $4 \mathrm{~h}$, after which they were successively immersed in xylene and $95 \%$ ethanol. To inhibit endogenous peroxidase activity, the lung sections were incubated with fresh $0.3 \%$ hydrogen peroxide in $100 \%$ methanol for $30 \mathrm{~min}$ at $37^{\circ} \mathrm{C}$. After washing the sections three times by PBS, antigen retrieval was performed by incubation with a $0.01 \mathrm{M}$ citrate buffer $(\mathrm{pH} 6.0)$ at $95-100{ }^{\circ} \mathrm{C}$ for 
15 min. The sections were then cooled to room temperature and washed with PBS, followed by incubation with bovine serum albumin solution for $30 \mathrm{~min}$, and then with an anti-p63 antibody (ab53039, Abcam; 1:300 dilution) at $37^{\circ} \mathrm{C}$ for $1 \mathrm{~h}$ and overnight at $4{ }^{\circ} \mathrm{C}$. Subsequently, the sections were washed three times with PBS and incubated with an HRP-conjugated goat anti-mouse secondary antibody (ab205718, Abcam) at room temperature for $45 \mathrm{~min}$. The sections were imaged using a $\times 40$ objective under a light microscope (CX43, Olympus, Tokyo, Japan).

\section{Protein digestion for mass spectrometry (MS) analysis}

Samples were lyophilized and then lysed in SDS solution (4\% SDS, $100 \mathrm{mM}$ Tris, pH 7.6). The protein solution from each sample was subjected to proteolytic digestion on 10-kDa filter (Pall Life Sciences, USA) using a filter-aided sample-preparation protocol, as described in detail elsewhere (33). The peptides in solution were transferred to a solid-phase extraction cartridge (MonoSpin C18, GL Sciences) for desalting and washing the samples. The peptides were dried by vacuum centrifugation for subsequent proteome analysis.

\section{Proteomic analysis}

All samples obtained as described above were analyzed with a Fusion mass spectrometer (Thermo Fisher Scientific) equipped with a Nanospray Flex source (Thermo Fisher Scientific). Data-dependent acquisition was performed using Xcalibur software in profile spectrum data type. All raw Xcalibur files acquired from the MS runs were analyzed using the default settings of Proteome Discoverer 2.2 (Thermo Scientific), with minor modifications. A detailed description of data analysis is provided in the Supplementary Information section.

\section{Lipid extraction for MS analysis}

Plasma samples were mixed with nine volumes of pre-cooled methanol: isopropanol solution (1:1). Then, the mixtures were left at room temperature for $10 \mathrm{~min}$ and placed at $-20^{\circ} \mathrm{C}$ overnight. After centrifuging at $14,000 \mathrm{rpm}$ for $20 \mathrm{~min}$, each supernatant was collected and stored at $-20^{\circ} \mathrm{C}$ for analysis. Both negative and positive electrospray-ionization modes were used to analyze the lipid extracts by triple-quadrupole MS (QTRAP 4000 and 6500; SCIEX, Framingham, MA, USA), as described (34).

\section{Lipidomic analysis}

Lipid identification was achieved by analyzing the MS data and using the bioinformatics tool, Lipid MS Predict (http://www.lipidmaps.org/). Peak extraction and identification based on the raw data were performed to ensure correct peak assignment. This process was carried out using Analyst software (version 1.6, SCIEX). Normalization was performed to obtain accurate relative intensities of the individual lipids. Multivariate statistical analysis and cluster analysis for lipids were performed using MetaboAnalyst software, version 3.0.

\section{Statistical analysis}

Statistical analysis was performed using GraphPad Prism 8.0 software (GraphPad, San Diego, CA, USA). One-way analysis of variance (ANOVA) was performed for multiple comparisons, whereas Student's $t$-test 
was applied for comparisons of two groups. $\mathrm{P}<0.05$ was defined as the threshold for statistical significance. The results are shown as the mean \pm standard deviation (SD).

\section{Results}

\section{MSC SLP attenuated mortality and weight loss induced by BLM}

Intratracheal BLM has been widely used to induce ALI within 1 week in mice (3). In this study, each mouse received $2.5 \mathrm{mg} / \mathrm{kg}$ BLM intratracheally. As shown in Fig. 1a, the mice were intratracheally administered PBS, BLM, or MSC SLP plus BLM on day 0, corresponding to the control, BLM, and MSC SLP groups, respectively. Peripheral blood, BALFs, and lung tissues were collected on day 7.

The survival status of the mice in each group was recorded daily, which showed that BLM led to higher mortality (approximately 20\%) on day 7, whereas co-treatment with MSC SLP significantly reduced the mortality ( $0 \%$ ) on day 7 (Fig. 1b). Furthermore, the mouse body weights decreased dramatically after BLM instillation, which was significantly reversed by MSC SLP (Fig. 1c). No visible weight loss was found in control group. These results demonstrated the contribution of MSC SLP to survival and weight maintenance in the mice, suggesting its potential for conferring a protective effect against ALI.

\section{MSC SLP attenuated BLM-induced alveolar injury}

H\&E staining demonstrated extensive morphological damage in BLM-instilled lungs, such as hemorrhaging, congestion, thickening of the alveolar walls, transparent membrane formation, and infiltration of inflammatory cells, especially neutrophils (Fig. 1d). In contrast, assessment of lung pathology revealed markedly more intact alveolar walls and decreased inflammation after MSC SLP treatment (Fig. 1d). Additionally, the lung-injury score (a relatively quantitative indicator) of MSC SLPinstilled mice was distinctly lower than that in BLM-instilled mice (Fig. 1e). BLM-induced alveolar septum thickening, alveolar-space broadening, and alveolar wall destruction were greatly counteracted by MSC SLP (Fig. 1f-h). No histological defects were visualized in the PBS-instilled lungs (Fig. $1 d-h$ ). The above results indicated that MSC SLP played a significant role in protecting alveoli from BLM-induced damage.

\section{MSC SLP inhibited apoptosis induced by BLM}

TUNEL assays were performed to estimate the number of apoptotic cells containing DNA fragments during the late stages of apoptosis (35). To clarify the effect of MSC SLP on apoptosis, we determined the degree of apoptosis in the lungs by TUNEL staining. TUNEL-positive epithelial cells dramatically increased in BLM-instilled lungs and remarkably restricted by MSC SLP (Fig. 2a). MSC SLP was competent in inhibiting apoptosis, which was possibly attributable to anti-apoptotic cytokines released by MSCs.

\section{MSC SLP alleviated inflammatory infiltration induced by BLM}


To determine the effect of MSC SLP on inflammatory infiltration, we assessed total protein levels, total cell numbers, and the profiles of inflammatory cells in BALFs. BLM instillation led to protein accumulation in BALFs and was remarkably blocked by MSC SLP (Fig. 2b). Moreover, BLM instillation significantly induced inflammatory cell infiltration, especially neutrophils, whereas MSC SLP significantly prevented lung inflammation (Fig. 2c, d). No statistically significant difference in inflammatory cell infiltration was found between the control and MSC SLP groups (Fig. 2c, d). To confirm this observation, the activity of MPO (a marker of neutrophilic aggregation) was evaluated in lung tissues to assess the level of neutrophils. BLM instillation caused a remarkable increased in the MPO levels, which was inhibited by MSC SLP (Fig. 2e).

\section{MSC SLP modulated the imbalance of Treg and Th17 cells}

Data from numerous studies have demonstrated that Treg and Th17 cells are associated with ALI and ARDS, in both humans and mice (36-38). Accordingly, we analyzed the balance of $\mathrm{CD} 4^{+} \mathrm{CD} 25^{+} \mathrm{Foxp} 3^{+}$ Treg cells and Th17 cells on day 7 by flow cytometry. BLM administration led to a reduction of CD $4^{+} T$ cells and an increased percentage of $\mathrm{CD} 4^{+} \mathrm{CD} 25^{+} \mathrm{Foxp}^{+}{ }^{+}$Treg cells (Fig. 2f, g), whereas MSC SLP stimulated $\mathrm{CD}^{+} \mathrm{T}$ cell differentiation and inhibited the expansion of Treg cells in lung tissues (Fig. $2 \mathrm{f}, \mathrm{g}$ ). Moreover, the number of Th17 cells in the blood was significantly attenuated after MSC SLP treatment (Fig. 2h).

\section{MSC SLP depressed IL-6 secretion induced by BLM}

$\mathrm{ALI}$ is an acute and inflammatory disorder involving the release of numerous cytokines. Thus, multiplex cytokine-detection technology was applied. Among all cytokines detected in the plasma, the IL- 6 concentration was elevated by BLM and efficiently mitigated by MSC SLP (Fig. 3a). To verify these findings, the levels of IL- 6 in plasma and BALFs were also measured by performing ELISAs. MSC SLP markedly neutralized IL-6 induction by BLM in both plasma and BALFs (Fig. 3b, c). ELISA analysis also showed that MSC SLP attenuated increased IL-1 $\beta$ production in BALFs (Fig. 3d). MSC SLP tended to lower the levels of IL-10, IL-13, and IL-23 in the plasma, although these differences were not statistically significant (Fig. 3a).

\section{MSC SLP activated p63 by inhibiting IL-6-p-STAT3 signaling}

p63 has been shown to support self-renewal, inhibit cell apoptosis, and help maintain homeostasis in epithelia (21, 23-25). In this study, RT-qPCR and western blot analysis revealed that p63 expression dramatically decreased in BLM-instilled mice and that MSC SLP restored p63 expression (Fig. 4a, b). In agreement, IHC showed that p63 was highly expressed in the basal layers of the airways, but was not expressed in the muscle layer of airways. p63 expression decreased in BLM-instilled mice, but partially recovered after MSC SLP treatment (Fig. 4c). Furthermore, immunofluorescence staining also showed that p63 expression was re-activated by MSC SLP (Fig. 5a-c). a-SMA, a marker of airway smooth muscle cells in the lungs, did not co-localize with p63 (Fig. 5a, e). 
Ki-67 (a proliferation marker) markedly co-localized with p63, especially in MSC SLP-instilled lungs (Fig. $5 \mathrm{c}-\mathrm{e}$ ), indicating that a considerable portion of $\mathrm{p} 63^{+}$cells was actively proliferating and repairing damage after MSC SLP instillation. Intensity profiles showed various degrees of co-localization between p63 and Ki-67 (Fig. 5d). To quantify the degree of co-localization between the fluorophores, Pearson's correlation coefficient (PCC) and Mander's overlap coefficient (MOC) (39) values were determined. Quantitative analysis verified that the p63 protein did not co-localize with a-SMA in the lungs, but was highly co-localized with Ki-67 (Fig. 5e).

Previous data showed that IL-6 regulates STAT3 signaling (40). Therefore, we subsequently detected STAT3 and activated STAT3 (p-STAT3) by western blotting. STAT3 was evidently activated to p-STAT3 in BLM-induced ALI (Fig. 6a, b). In contrast, MSC SLP significantly inhibited STAT3 phosphorylation (Fig. 6a, b). Considering that IL- 6 appeared to promote STAT3 phosphorylation in basal cells of the airways and previous data showed that the IL-6-p-STAT3 pathway regulated p63 isoform expression in keratinocytes $(40,41)$, we hypothesized that a sharp rise of IL-6 boosted STAT3 phosphorylation and then restrained p63 expression in BLM-induced ALI. Our results suggested that MSC SLP activated p63 by inhibiting the IL-6-p-STAT3 pathway.

\section{Intratracheal rh IL-6 reduced p63 expression}

To clarify the role of IL-6, we instilled rh IL-6 into the airways of mice. ELISAs demonstrated that rh IL-6 was enriched in the lung tissues on day 1 , after which it had been absorbed and removed on day 2 after rh IL-6 instillation (Fig. 6c). Consistently, rh IL-6 administration promoted STAT3 phosphorylation and decreased p63 expression (Fig. 6d-f). In the presence of sufficient exogenous IL-6, MSC SLP no longer reactivated p63 expression after rh IL- 6 instillation. These results suggested that MSC SLP alleviated ALI by inhibiting inflammatory cell recruitment and reducing IL-6 production, without blocking IL- 6 function.

\section{MSC SLP increased JAG2 expression}

To clarify the mechanism whereby MSC SLP alleviated ALI, we tested various target genes of p63, especially those linked to basal cell function. In this study, the mRNA and protein levels of JAG2 were both significantly down-regulated by BLM (Fig. 7a-c), whereas MSC SLP greatly increased JAG2 expression (Fig. 7a-c). Administration of rh IL-6 reduced JAG2 expression, which was not reversed by MSC SLP (Fig. 7d, e). A schematic model whereby MSC SLP alleviated BLM-induced ALI is proposed in Fig. $7 \mathrm{f}$.

\section{MSC SLP regulated the lipid profile in plasma}

To obtain an overview of the alterations in the plasma lipid profiles induced by BLM and MSC SLP, we determined the levels of 373 individual lipid species, including phosphoglycerolipids, sphingolipids, neutral lipids, and other lipids (Fig. 8a). Principal component analysis revealed apparent separations between the control, BLM-instilled, and MSC SLP-instilled mice on day 7 (Fig. 8b). A heatmap was generated, which demonstrated the significant differences found in the three groups (Fig. 8c). Each row 
and column shows individual lipids in each sample type. We performed relative quantifications of the major lipid classes (Fig. 8d). PC species were the most prominent lipids in the plasma, followed by lysoPC. The overall content of phosphatidylserine (PS) was significantly reduced by BLM and greatly increased by MSC SLP (Fig. 8d). Among these, the levels of PS 20:3/22:6 and PS 38:3 changed most dramatically. MSC SLP treatment greatly increased the levels of PS 20:3/22:6 and PS 38:3 reduced by BLM (Fig. 8e). Moreover, among other lipid subclasses, 12 lipids were altered considerably (Fig. 8f).

\section{Discussion}

Preclinical studies and currently ongoing clinical trials have shown that MSC administration has the potential to become an effective therapeutic strategy for treating $\operatorname{ALI}(10,11,14,42-44)$. MSCs provide protective roles against ALI either by differentiating into alveolar epithelial cells after damage, or by secreting soluble factors such as keratinocyte growth factors, anti-inflammatory cytokines, and extracellular vesicles $(14,43,44)$. However, limitations in terms of delivery and risks of instability in humans are both concerns, especially considering the danger of iatrogenic tumor formation (14). The secretome of MSCs, composed of extracellular vesicles and released bioactive molecules, is a viable alternative cell-free therapy. MSC-conditioned media, containing proteins, cytokines, and chemokines released by MSCs, can enhance calvarial bone regeneration in rats (10) and induce neutrophil apoptosis to attenuate ALI (45). The secretome of MSCs can be purified and freeze-dried into lyophilized powder to achieve large-scale production, easier storage, and stable bioactivity for a long time (46). To delineate the bioactive ingredients in lyophilized powder from MSCs, liquid chromatography (LC)-tandem MS technology was performed, and numerous active biomolecules potentially involved are shown in Supplementary Table 1.

In this study, MSC SLP was found to reduce mortality, help maintain alveolar morphological structures, reduce alveolar inflammation, and inhibit epithelial cell apoptosis in BLM-induced ALI. The induction of p63 expression by MSC SLP was the most important finding of our study. Data from numerous studies have demonstrated that p63, a homolog of p53, is vital for the normal development and homeostasis of epithelial tissues, in both humans and mice. For instance, heterozygous mutations in human p63 drives several developmental defects and disorders, especially skin abnormalities (47-49). In animal studies, p63 knockout led to severe anomalies in the development of epithelia and their derivatives, and even death at birth $(25,50)$. p63 is also widely recognized as being involved in tumorigenesis, especially epithelial tumors, such as those associated with prostate, bladder, and colorectal cancer $(26,27)$. In addition, p63 is a specific marker of basal cells and serves a vital role in helping maintain self-renewal potential and inhibit apoptosis in various epithelial cells, including those in the mouse trachea and human airways $(21,22,24)$. In this study, we showed that MSC SLP re-activated p63 expression and suppressed epithelial cell apoptosis in BLM-induced ALI, indicating that p63 plays a crucial role in ALI development. Immunofluorescence staining against p63 and Ki-67 demonstrated the presence of proliferating $163^{+}$cells. Thus, MSC SLP was verified to activate p63 expression to improve the proliferative capacity and inhibit apoptosis in progenitor cells. 
IL-6 is a pleiotropic cytokine that is produced in response to various inflammatory stimuli and that is associated with multiple inflammatory disorders of the lungs (15-18). In BLM-treated mice, IL-6 promoted inflammatory infiltration at an early inflammatory stage (20). In this study, on day 7 , the sharp increase of IL- 6 production in response to BLM stimulation was markedly repressed by MSC SLP. Moreover, IL-6 appeared to regulate 063 expression by promoting STAT3 phosphorylation in mice. Previous data revealed that the IL-6-p-STAT3 pathway was activated during $\mathrm{SO}_{2}$-induced airway injury in mice and that STAT3 activation in basal cells regulated ciliogenesis through tyrosine phosphorylation (40). IL-6-p-STAT3-pathway activation modulated expression of the p63 isoform in keratinocytes during regeneration associated with wound healing(41). In this study, we found that hyperphosphorylation of STAT3, attributed to excessive IL-6 secretion, inhibited p63 expression in BLM-induced ALI, whereas MSC SLP increased p63 expression by suppressing excessive activation of IL- 6 and p-STAT3.

$\mathrm{CD} 4^{+} \mathrm{CD} 25^{+} \mathrm{Foxp}^{+}$Treg cells negatively regulate immune and inflammatory responses by cell-contactdependent suppression and secretion of inhibitory cytokines, such as IL-10 and transforming growth factor (TGF)- $\beta$, which prevents chronic immunopathology after pathogen clearance (51). However, studies of the roles of Treg cells in different diseases seemed contradictory (52). Under many inflammatory conditions, a sudden increase of Treg cells without beneficial functions can occur (52). For instance, patients with ALI or ARDS often show increased levels of Treg cells, which have been associated with mortality of patients with ARDS $(37,38)$. In this study, BLM increased the percentage of CD ${ }^{+} \mathrm{CD} 25^{+} \mathrm{Foxp}^{+}$Treg cells in the lungs, which was reversed by MSC SLP. Apparently, the surge in Treg cell production after BLM instillation could not control inflammatory injury. Th17 cells have opposite roles, when compared to Treg cells, and present a subset of pro-inflammatory cells (51). In ARDS, Th17 cells enhanced the accumulation of pro-inflammatory cytokines and amplified inflammatory responses $(37,38)$. Here, we found that MSC SLP dramatically attenuated the increase of Th17 cells in the blood. Furthermore, accumulating evidence has revealed that IL- 6 can synergize with TGF- $\beta$ to initiate Th17 polarization by promoting STAT3 phosphorylation(53-55). Consistently, we found that MSC SLP strongly inhibited IL-6 production and STAT3 phosphorylation. Therefore, we hypothesize that MSC SLP restrained Th17 cell expansion by inhibiting IL-6-induced STAT3 phosphorylation, which eventually alleviated inflammatory injury.

p63-mediated target gene expression confers stem cell properties, such as the proliferative capacity and differentiation of basal cells $(23,24)$. Previous findings revealed that the expression of JAG2 (a Notch ligand) was directly regulated by p63 in epithelia, which was associated with progenitor cell differentiation and restrained inflammation. In vitro, JAG2 was strongly expressed on the surface of $p 63^{+}$ adult airway progenitor cells (56). In vivo, JAG2 expression was enhanced by $\mathrm{p} 63$ and was required for thymic development (57). JAG2-knockout mice presented with limb defects and thymic underdevelopment, and some even died at birth (58). Increased JAG2 expression in p63 ${ }^{+}$airway progenitor cells could activate Notch 3 signaling in neighboring cells, ultimately promoting $163^{+}$cell expansion, the transition to early epithelial progenitors, and later to daughter cell fate selection $(56,59$, 60). Moreover, JAG2 inhibited monocytic recruitment by reducing the expression of inflammation-related 
genes in human non-small cell lung cancer cells (61). Consistently, we observed that JAG2 expression was down-regulated by reduced p63 signaling in BLM-instilled lungs and was up-regulated by MSC SLP treatment. Therefore, we postulate that MSC SLP activated the p63-JAG2 pathway, which alleviated inflammatory cell recruitment and promoted the repair of damaged epithelial tissue.

In terms of lipidomics, previous reports have shown the anti-inflammatory effects of PS on several inflammation-related diseases, such as rheumatoid arthritis $(62,63)$ and myocardial infarction (64). PS also reduced the secretion of pro-inflammatory cytokines, including IL-1 $\beta$ and IL-6, both in vivo and in vitro $(62,63)$. Subsequent studies were performed to examine the associated mechanisms. For example, macrophages were found to specifically recognize PS molecules expressed on the surface of apoptotic cells, which was crucial for macrophage-induced anti-inflammatory responses, including reduced secretion of pro-inflammatory cytokines (IL-1 $\beta, \mathrm{IL}-6$, and TNF- $\alpha$ ) and increased secretion of antiinflammatory cytokines (TGF- $\beta$ and IL-10) $(65,66)$. Exogenous PS also mimicked apoptotic cells and promoted a phenotypic switch from inflammatory M1 macrophages to anti-inflammatory M2 macrophages $(65,66)$. In this study, MSC SLP significantly reversed PS down-regulation in BLM-instilled mice, thereby enhancing macrophage-induced anti-inflammatory responses and restraining IL-1 $\beta$ and IL- 6 production, and eventually promoting the resolution of inflammation and lung injury repair. Moreover, PS can be readily extracted from soybeans and administered orally in humans(67). Thus, we regard PS supplementation as a potential therapeutic strategy for treating ALI.

Emerging evidence has illustrated that lyophilization does not disturb the structure and integrity of extracellular vesicles (68) and does not weaken the stability of proteins and lipid components (46). Airway administration of MSC SLP ensured that it was relatively enriched at sites of damage in the lungs, suggesting that MSC SLP holds substantial therapeutic promise for clinical applications.

\section{Conclusions}

We demonstrated that MSC SLP depressed signaling through the IL-6-p-STAT3 pathway and then activated p63-JAG2 signaling to promote epithelial cell proliferation, inhibit cell apoptosis, reduce inflammatory cell recruitment, and ameliorate ALI. Inhibition of the IL-6-p-STAT3-signaling pathway also restricted Th17 cell polarization. Moreover, MSC SLP modulated lipid profiles, especially those of PS species. The results of this study shed light on the mechanisms of BLM-induced ALI and suggest that treatment with MSC SLP offers promise as a therapeutic approach.

\section{List Of Abbreviations}

a-SMA, alpha smooth muscle actin; ALI, acute lung injury; ANOVA, analysis of variance; APC, allophycocyanin; ARDS, acute respiratory distress syndrome; BALF, bronchoalveolar lavage fluid; BCA, bicinchoninic acid; BLM, bleomycin; CD, cluster of differentiation; CST, Cell Signaling Technology; DI, destructive index; ELISA, enzyme-linked immunosorbent assay; FITC, fluorescein isothiocyanate; Foxp3, forkhead box P3; GM-CSF, granulocyte-macrophage colony-stimulating factor; $\mathrm{H} \& \mathrm{E}$, hematoxylin and 
eosin; HRP, horseradish peroxidase; IHC, immunohistochemistry; IFN, interferon; IL, interleukin; JAG2, jagged 2; Krt14, cytokeratin 14; Krt5, cytokeratin 5; LC, liquid chromatography; MAST, mean alveolar septal thickness; MLI, mean linear intercept; MS, mass spectrometry; MOC, Mander's overlap coefficient; MSC, mesenchymal stem cell; MPO, myeloperoxidase; P1, passage 1; p63, tumor protein 63; p-STAT, phosphorylated signal transducer and activator of transcription; PBS, phosphate-buffered saline; PC, phosphatidylcholine; PCC, Pearson's correlation coefficient; PE, phosphatidylethanolamine; PS, phosphatidylserine; rh, recombinant human; SD, standard deviation; SDS, sodium dodecyl sulfate; SLP, supernatant lyophilized powder; TGF, transforming growth factor; TH17, T helper 17; TNF, tumor necrosis factor; Treg, regulatory T cell; TUNEL, terminal deoxynucleotidyl transferase dUTP nick-end labeling

\section{Declarations}

\section{Author Contributions}

Peng, Chang, Wu, W.Zhu, and Tong contributed equally to this study. Dr. Zhou, H.Li and Song had full access to all the data in the study and takes responsibility for the integrity of the data and accuracy of the data analysis. Study concept and design: Zhou, H.Li, and Song. Experiments and data collection: Peng, Chang, Wu, W.Zhu, Tong, G.Zhang, Wang, Liu, X.Zhu, Cheng, Y.Li, Chen, Weng, Liu, H.Zhang, and Su. Data interpretation and statistical analysis: Peng, Chang, Wu, W.Zhu, and Tong. Drafting of the manuscript: Peng and Zhou. Study supervision: Zhou, H.Li and Song.

\section{Funding}

This work was supported by National Science \& Technology Major Project "Key New Drug Creation and Manufacturing Program" (2018ZX09201002-006), National Natural Science Foundation of China (81770039, 81570028, and 81770075), National key R\&D plan (2016YFC1304104), Zhongshan Hospital Clinical Research Foundation (2019ZSGG15), Shanghai Municipal Key Clinical Specialty (shslczdzk02201), Science and Technology Commission of Shanghai Municipality (20XD1401200).

\section{Availability of data and materials}

All data needed to evaluate the conclusions in the paper are present in the paper and/or the Supplementary Materials. Additional data available from authors upon request.

\section{Ethics approval and consent to participate}

The experimental protocols were approved by the Animal Care and Use Committee of Zhongshan Hospital at Fudan University.

\section{Consent for publication}

Not applicable.

\section{Competing interests}


The authors have declared no competing interests.

\section{Authors' information}

${ }^{1}$ Department of Pulmonary and Critical Care Medicine, Shanghai Respiratory Research Institute, Zhongshan Hospital, Fudan University, Shanghai 200032, China.

${ }^{2}$ Department of Pulmonary and Critical Care Medicine, Shanghai East Hospital, Tongji University School of Medicine, Shanghai 200120, China.

${ }^{3}$ Yunnan Province Stem cell Bank, Yunnan, Kunming 650101, China.

${ }^{4}$ Department of Pulmonary and Critical Care Medicine, Shanghai Pulmonary Hospital, Tongji University School of Medicine, Shanghai 200433, China.

${ }^{5}$ Institute of Interdisciplinary Integrative Medicine Research, Shanghai University of Traditional Chinese Medicine, Shanghai 201203, China.

${ }^{6}$ Center of Emergency \& Intensive Care Unit, Jinshan Hospital, Fudan University, Shanghai 200540, China;

7 Public Translational Platform for Cell Therapy, Yangtze Delta Region Institute of Tsinghua University, Zhejiang, Hangzhou 311200, China.

\section{References}

1. Wheeler AP, Bernard GR. Acute lung injury and the acute respiratory distress syndrome: a clinical review. Lancet. 2007;369(9572):1553-64.

2. Adamson IY. Pulmonary toxicity of bleomycin. Environ Health Perspect. 1976;16:119-26.

3. Matute-Bello G, Frevert CW, Martin TR. Animal models of acute lung injury. Am J Physiol Lung Cell Mol Physiol. 2008;295(3):L379-99.

4. Shi Y, Wang Y, Li Q, Liu K, Hou J, Shao C, et al. Immunoregulatory mechanisms of mesenchymal stem and stromal cells in inflammatory diseases. Nat Rev Nephrol. 2018;14(8):493-507.

5. da Silva Meirelles L, Chagastelles PC, Nardi NB. Mesenchymal stem cells reside in virtually all postnatal organs and tissues. J Cell Sci. 2006;119(Pt 11):2204-13.

6. Zuk PA, Zhu M, Ashjian P, De Ugarte DA, Huang JI, Mizuno H, et al. Human adipose tissue is a source of multipotent stem cells. Mol Biol Cell. 2002;13(12):4279-95.

7. Sabatini F, Petecchia L, Tavian M, Jodon de Villeroche V, Rossi GA, Brouty-Boye D. Human bronchial fibroblasts exhibit a mesenchymal stem cell phenotype and multilineage differentiating potentialities. Lab Invest. 2005;85(8):962-71.

8. He Q, Wan C, Li G. Concise review: multipotent mesenchymal stromal cells in blood. Stem Cells. 2007;25(1):69-77. 
9. Romanov YA, Svintsitskaya VA, Smirnov VN. Searching for alternative sources of postnatal human mesenchymal stem cells: candidate MSC-like cells from umbilical cord. Stem Cells. 2003;21(1):10510.

10. Osugi M, Katagiri W, Yoshimi R, Inukai T, Hibi H, Ueda M. Conditioned media from mesenchymal stem cells enhanced bone regeneration in rat calvarial bone defects. Tissue Eng Part A. 2012;18(1314):1479-89.

11. Monsel A, Zhu YG, Gudapati V, Lim H, Lee JW. Mesenchymal stem cell derived secretome and extracellular vesicles for acute lung injury and other inflammatory lung diseases. Expert Opin Biol Ther. 2016;16(7):859-71.

12. Wilson JG, Liu KD, Zhuo H, Caballero L, McMillan M, Fang X, et al. Mesenchymal stem (stromal) cells for treatment of ARDS: a phase 1 clinical trial. Lancet Respir Med. 2015;3(1):24-32.

13. Lee JW, Fang X, Krasnodembskaya A, Howard JP, Matthay MA. Concise review: Mesenchymal stem cells for acute lung injury: role of paracrine soluble factors. Stem Cells. 2011;29(6):913-9.

14. Walter J, Ware LB, Matthay MA. Mesenchymal stem cells: mechanisms of potential therapeutic benefit in ARDS and sepsis. Lancet Respir Med. 2014;2(12):1016-26.

15. Park WY, Goodman RB, Steinberg KP, Ruzinski JT, Radella F, 2nd, Park DR, et al. Cytokine balance in the lungs of patients with acute respiratory distress syndrome. Am J Respir Crit Care Med. 2001;164(10 Pt 1):1896-903.

16. Zhang H, Neuhofer P, Song L, Rabe B, Lesina M, Kurkowski MU, et al. IL-6 trans-signaling promotes pancreatitis-associated lung injury and lethality. J Clin Invest. 2013;123(3):1019-31.

17. Klein CL, Hoke TS, Fang WF, Altmann CJ, Douglas IS, Faubel S. Interleukin-6 mediates lung injury following ischemic acute kidney injury or bilateral nephrectomy. Kidney Int. 2008;74(7):901-9.

18. Goldman JL, Sammani S, Kempf C, Saadat L, Letsiou E, Wang T, et al. Pleiotropic effects of interleukin-6 in a "two-hit" murine model of acute respiratory distress syndrome. Pulm Circ. 2014;4(2):280-8.

19. Saito F, Tasaka S, Inoue K, Miyamoto K, Nakano Y, Ogawa Y, et al. Role of interleukin-6 in bleomycininduced lung inflammatory changes in mice. Am J Respir Cell Mol Biol. 2008;38(5):566-71.

20. Kobayashi T, Tanaka K, Fujita T, Umezawa H, Amano H, Yoshioka K, et al. Bidirectional role of IL-6 signal in pathogenesis of lung fibrosis. Respir Res. 2015;16:99.

21. Rock JR, Onaitis MW, Rawlins EL, Lu Y, Clark CP, Xue Y, et al. Basal cells as stem cells of the mouse trachea and human airway epithelium. Proc Natl Acad Sci U S A. 2009;106(31):12771-5.

22. Zuo W, Zhang T, Wu DZ, Guan SP, Liew AA, Yamamoto Y, et al. p63(+)Krt5(+) distal airway stem cells are essential for lung regeneration. Nature. 2015;517(7536):616-20.

23. Senoo M, Pinto F, Crum CP, McKeon F. p63 Is essential for the proliferative potential of stem cells in stratified epithelia. Cell. 2007;129(3):523-36.

24. Melino G, Memmi EM, Pelicci PG, Bernassola F. Maintaining epithelial stemness with p63. Sci Signal. 2015;8(387):re9. 
25. Yang A, Schweitzer R, Sun D, Kaghad M, Walker N, Bronson RT, et al. p63 is essential for regenerative proliferation in limb, craniofacial and epithelial development. Nature. 1999;398(6729):714-8.

26. Pignon JC, Grisanzio C, Geng Y, Song J, Shivdasani RA, Signoretti S. p63-expressing cells are the stem cells of developing prostate, bladder, and colorectal epithelia. Proc Natl Acad Sci U S A. 2013;110(20):8105-10.

27. Moses MA, George AL, Sakakibara N, Mahmood K, Ponnamperuma RM, King KE, et al. Molecular Mechanisms of p63-Mediated Squamous Cancer Pathogenesis. Int J Mol Sci. 2019;20(14).

28. Fadok VA, Bratton DL, Rose DM, Pearson A, Ezekewitz RA, Henson PM. A receptor for phosphatidylserine-specific clearance of apoptotic cells. Nature. 2000;405(6782):85-90.

29. Ridgway ND. The role of phosphatidylcholine and choline metabolites to cell proliferation and survival. Crit Rev Biochem Mol Biol. 2013;48(1):20-38.

30. Harayama T, Eto M, Shindou H, Kita Y, Otsubo E, Hishikawa D, et al. Lysophospholipid acyltransferases mediate phosphatidylcholine diversification to achieve the physical properties required in vivo. Cell Metab. 2014;20(2):295-305.

31. Nagase T, Uozumi N, Ishii S, Kita Y, Yamamoto H, Ohga E, et al. A pivotal role of cytosolic phospholipase A(2) in bleomycin-induced pulmonary fibrosis. Nat Med. 2002;8(5):480-4.

32. Saito K, Tanaka N, Ikari J, Suzuki M, Anazawa R, Abe M, et al. Comprehensive lipid profiling of bleomycin-induced lung injury. J Appl Toxicol. 2019;39(4):658-71.

33. Nielsen PA, Olsen JV, Podtelejnikov AV, Andersen JR, Mann M, Wisniewski JR. Proteomic mapping of brain plasma membrane proteins. Mol Cell Proteomics. 2005;4(4):402-8.

34. Han X, Gross RW. Shotgun lipidomics: electrospray ionization mass spectrometric analysis and quantitation of cellular lipidomes directly from crude extracts of biological samples. Mass Spectrom Rev. 2005;24(3):367-412.

35. Short B. TUNEL vision spots apoptotic cells. J Cell Biol. 2015;208(1):7.

36. Wang $L$, Wang $X$, Tong $L$, Wang J, Dou M, Ji S, et al. Recovery from acute lung injury can be regulated via modulation of regulatory T cells and Th17 cells. Scand J Immunol. 2018;88(5):e12715.

37. Yu ZX, Ji MS, Yan J, Cai Y, Liu J, Yang HF, et al. The ratio of Th17/Treg cells as a risk indicator in early acute respiratory distress syndrome. Crit Care. 2015;19:82.

38. Adamzik M, Broll J, Steinmann J, Westendorf AM, Rehfeld I, Kreissig C, et al. An increased alveolar $\mathrm{CD} 4+\mathrm{CD} 25+$ Foxp3 + T-regulatory cell ratio in acute respiratory distress syndrome is associated with increased 30-day mortality. Intensive Care Med. 2013;39(10):1743-51.

39. Adler J, Parmryd I. Quantifying colocalization by correlation: the Pearson correlation coefficient is superior to the Mander's overlap coefficient. Cytometry A. 2010;77(8):733-42.

40. Tadokoro T, Wang Y, Barak LS, Bai Y, Randell SH, Hogan BL. IL-6/STAT3 promotes regeneration of airway ciliated cells from basal stem cells. Proc Natl Acad Sci U S A. 2014;111(35):E3641-9.

41. Nelson AM, Katseff AS, Ratliff TS, Garza LA. Interleukin 6 and STAT3 regulate p63 isoform expression in keratinocytes during regeneration. Exp Dermatol. 2016;25(2):155-7. 
42. Hao Q, Zhu YG, Monsel A, Gennai S, Lee T, Xu F, et al. Study of Bone Marrow and Embryonic Stem Cell-Derived Human Mesenchymal Stem Cells for Treatment of Escherichia coli Endotoxin-Induced Acute Lung Injury in Mice. Stem Cells Transl Med. 2015;4(7):832-40.

43. Park J, Kim S, Lim H, Liu A, Hu S, Lee J, et al. Therapeutic effects of human mesenchymal stem cell microvesicles in an ex vivo perfused human lung injured with severe E. coli pneumonia. Thorax. 2019;74(1):43-50.

44. Ferreira JR, Teixeira GQ, Santos SG, Barbosa MA, Almeida-Porada G, Goncalves RM. Mesenchymal Stromal Cell Secretome: Influencing Therapeutic Potential by Cellular Pre-conditioning. Front Immunol. 2018;9:2837.

45. Hou Z, Wu Q, Sun X, Chen H, Li Y, Zhang Y, et al. Wnt/Fgf crosstalk is required for the specification of basal cells in the mouse trachea. Development. 2019;146(3).

46. Bari E, Perteghella S, Di Silvestre D, Sorlini M, Catenacci L, Sorrenti M, et al. Pilot Production of Mesenchymal Stem/Stromal Freeze-Dried Secretome for Cell-Free Regenerative Nanomedicine: A Validated GMP-Compliant Process. Cells. 2018;7(11).

47. Lin-Shiao E, Lan Y, Welzenbach J, Alexander KA, Zhang Z, Knapp M, et al. p63 establishes epithelial enhancers at critical craniofacial development genes. Sci Adv. 2019;5(5):eaaw0946.

48. Soares E, Xu Q, Li Q, Qu J, Zheng Y, Raeven HHM, et al. Single-cell RNA-seq identifies a reversible mesodermal activation in abnormally specified epithelia of p63 EEC syndrome. Proc Natl Acad Sci U S A. 2019;116(35):17361-70.

49. Soares E, Zhou H. Master regulatory role of p63 in epidermal development and disease. Cell Mol Life Sci. 2018;75(7):1179-90.

50. Santos-Pereira JM, Gallardo-Fuentes L, Neto A, Acemel RD, Tena JJ. Pioneer and repressive functions of p63 during zebrafish embryonic ectoderm specification. Nat Commun. 2019;10(1):3049.

51. Noack M, Miossec P. Th17 and regulatory T cell balance in autoimmune and inflammatory diseases. Autoimmun Rev. 2014;13(6):668-77.

52. Buckner $\mathrm{JH}$. Mechanisms of impaired regulation by CD4(+)CD25(+)FOXP3(+) regulatory T cells in human autoimmune diseases. Nat Rev Immunol. 2010;10(12):849-59.

53. Zhou L, Ivanov, II, Spolski R, Min R, Shenderov K, Egawa T, et al. IL-6 programs T(H)-17 cell differentiation by promoting sequential engagement of the IL-21 and IL-23 pathways. Nat Immunol. 2007;8(9):967-74.

54. Ma CS, Chew GY, Simpson N, Priyadarshi A, Wong M, Grimbacher B, et al. Deficiency of Th17 cells in hyper IgE syndrome due to mutations in STAT3. J Exp Med. 2008;205(7):1551-7.

55. Renner ED, Rylaarsdam S, Anover-Sombke S, Rack AL, Reichenbach J, Carey JC, et al. Novel signal transducer and activator of transcription 3 (STAT3) mutations, reduced $\mathrm{T}(\mathrm{H}) 17$ cell numbers, and variably defective STAT3 phosphorylation in hyper-IgE syndrome. J Allergy Clin Immunol. 2008;122(1):181-7.

56. Mori M, Mahoney JE, Stupnikov MR, Paez-Cortez JR, Szymaniak AD, Varelas X, et al. Notch3-Jagged signaling controls the pool of undifferentiated airway progenitors. Development. 2015;142(2):258-67. 
57. Candi E, Rufini A, Terrinoni A, Giamboi-Miraglia A, Lena AM, Mantovani R, et al. DeltaNp63 regulates thymic development through enhanced expression of FgfR2 and Jag2. Proc Natl Acad Sci U S A. 2007;104(29):11999-2004.

58. Jiang R, Lan Y, Chapman HD, Shawber C, Norton CR, Serreze DV, et al. Defects in limb, craniofacial, and thymic development in Jagged2 mutant mice. Genes Dev. 1998;12(7):1046-57.

59. Rock JR, Gao X, Xue Y, Randell SH, Kong YY, Hogan BL. Notch-dependent differentiation of adult airway basal stem cells. Cell Stem Cell. 2011;8(6):639-48.

60. Stupnikov MR, Yang Y, Mori M, Lu J, Cardoso WV. Jagged and Delta-like ligands control distinct events during airway progenitor cell differentiation. Elife. 2019;8.

61. Choi K, Ahn YH, Gibbons DL, Tran HT, Creighton CJ, Girard L, et al. Distinct biological roles for the notch ligands Jagged-1 and Jagged-2. J Biol Chem. 2009;284(26):17766-74.

62. Ma HM, Wu Z, Nakanishi H. Phosphatidylserine-containing liposomes suppress inflammatory bone loss by ameliorating the cytokine imbalance provoked by infiltrated macrophages. Lab Invest. 2011;91(6):921-31.

63. Yeom M, Hahm DH, Sur BJ, Han JJ, Lee HJ, Yang HI, et al. Phosphatidylserine inhibits inflammatory responses in interleukin-1 beta-stimulated fibroblast-like synoviocytes and alleviates carrageenaninduced arthritis in rat. Nutr Res. 2013;33(3):242-50.

64. Harel-Adar T, Ben Mordechai T, Amsalem Y, Feinberg MS, Leor J, Cohen S. Modulation of cardiac macrophages by phosphatidylserine-presenting liposomes improves infarct repair. Proc Natl Acad Sci U S A. 2011;108(5):1827-32.

65. Ravichandran KS, Lorenz U. Engulfment of apoptotic cells: signals for a good meal. Nat Rev Immunol. 2007;7(12):964-74.

66. Nagata S, Hanayama R, Kawane K. Autoimmunity and the clearance of dead cells. Cell. 2010;140(5):619-30.

67. Wang H, Yuan Q, Niu M, Zhang W, Wen L, Fu H, et al. Transcriptional regulation of P63 on the apoptosis of male germ cells and three stages of spermatogenesis in mice. Cell Death Dis. 2018;9(2):76.

68. Antes TJ, Middleton RC, Luther KM, ljichi T, Peck KA, Liu WJ, et al. Targeting extracellular vesicles to injured tissue using membrane cloaking and surface display. J Nanobiotechnology. 2018;16(1):61.

\section{Figures}


A

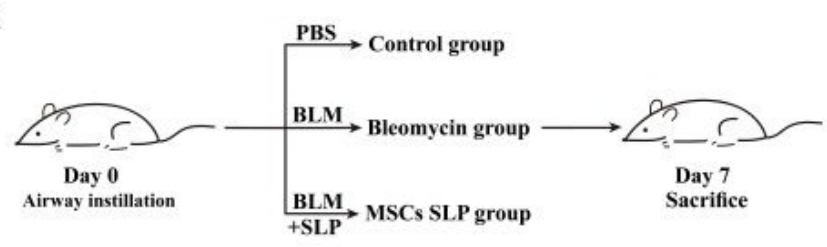

D

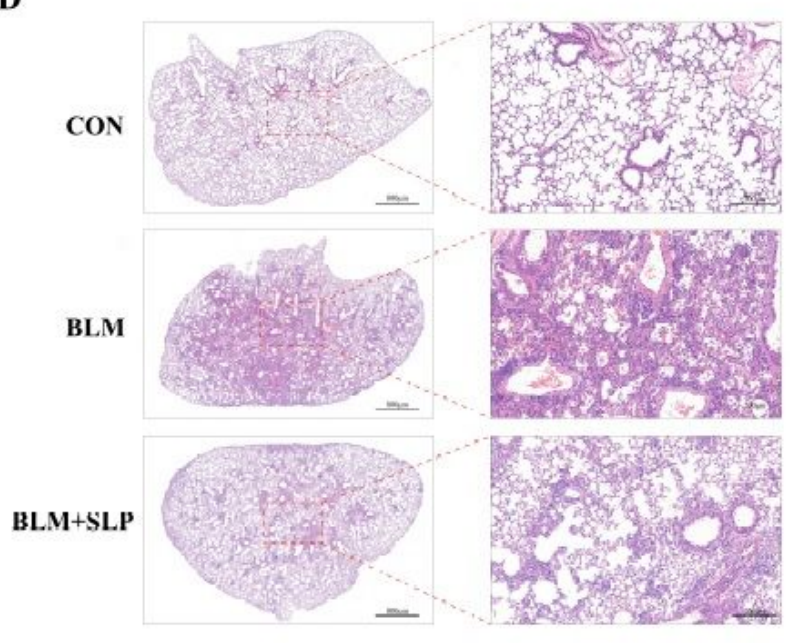

B

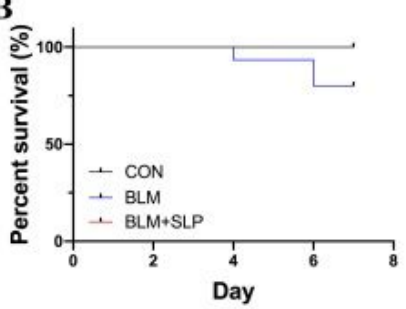

$\mathbf{E}$
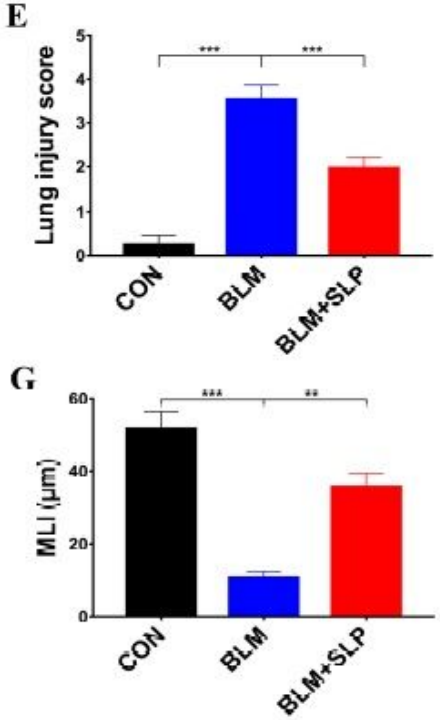

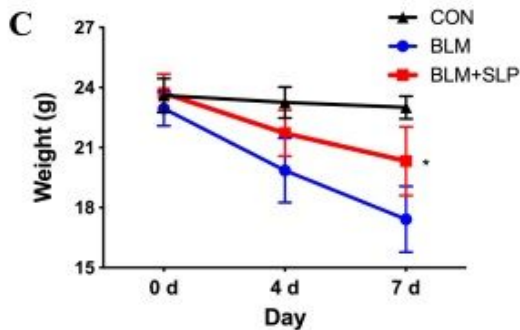

$\mathbf{F}$

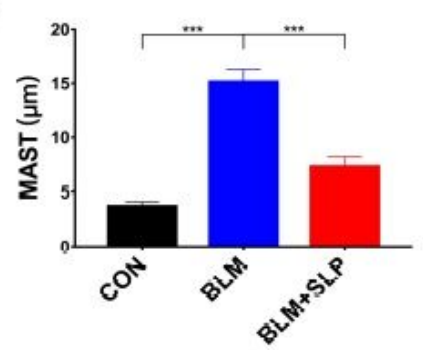

H

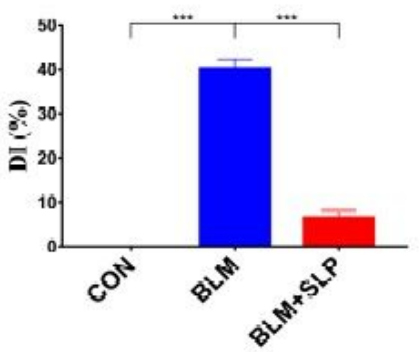

\section{Figure 1}

MSC SLP attenuated BLM-induced ALI in vivo. a Scheme representation of the mouse model established in this study. PBS, BLM $(2.5 \mathrm{mg} / \mathrm{kg})$, or BLM $(2.5 \mathrm{mg} / \mathrm{kg})$ plus MSC SLP $(50 \mathrm{mg} / \mathrm{kg})$ was intratracheally delivered to mice. b Survival curves. c Mouse weights on days 0,4 , and 7. d H\&E staining. e-h Quantitative analysis of lung damage as assessed histopathologically. e Lung injury score. $f$ Mean alveolar septal thickness (MAST). g Mean linear intercept (MLI). h Destructive index (DI). Ten fields were randomly selected for scoring. $\mathrm{N}=6-8$ in each group. The data shown are presented as the mean $\pm \mathrm{SD}$, and statistical differences were assessed by performing one-way ANOVA. ${ }^{*} \mathrm{P}<0.05$; ${ }^{\star \star} \mathrm{P}<0.01$; ${ }^{* \star} \mathrm{P}<$ 0.001 
A

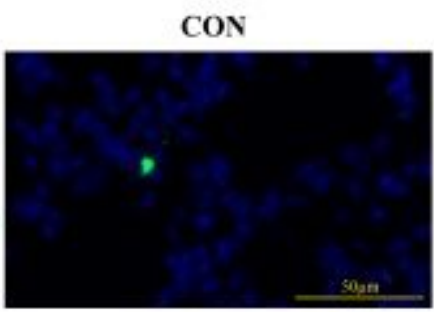

B

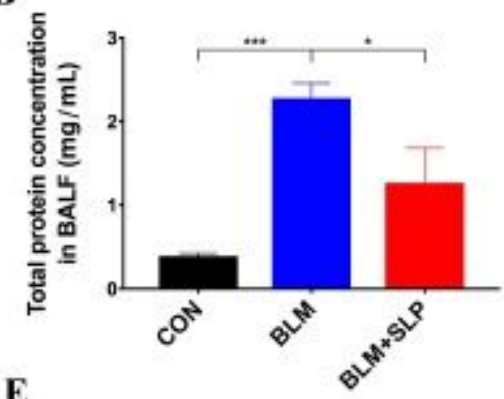

E

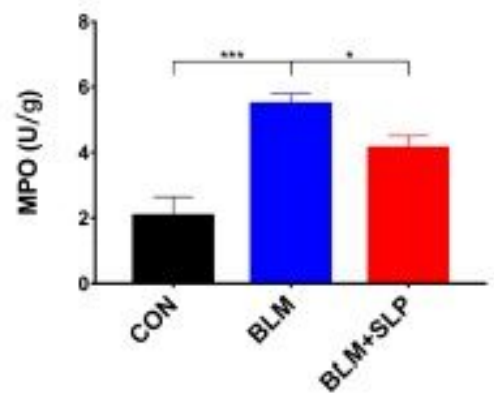

BLM

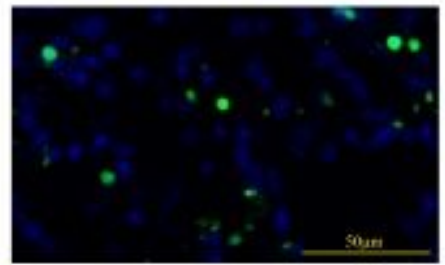

C
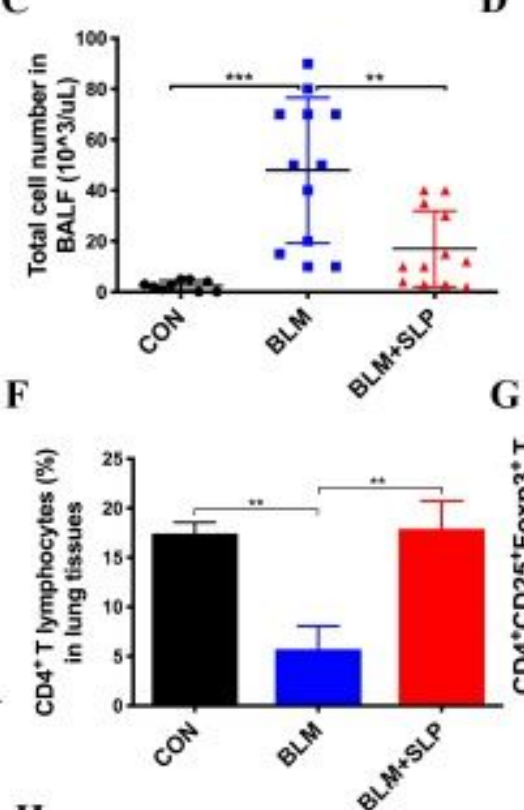

H

D
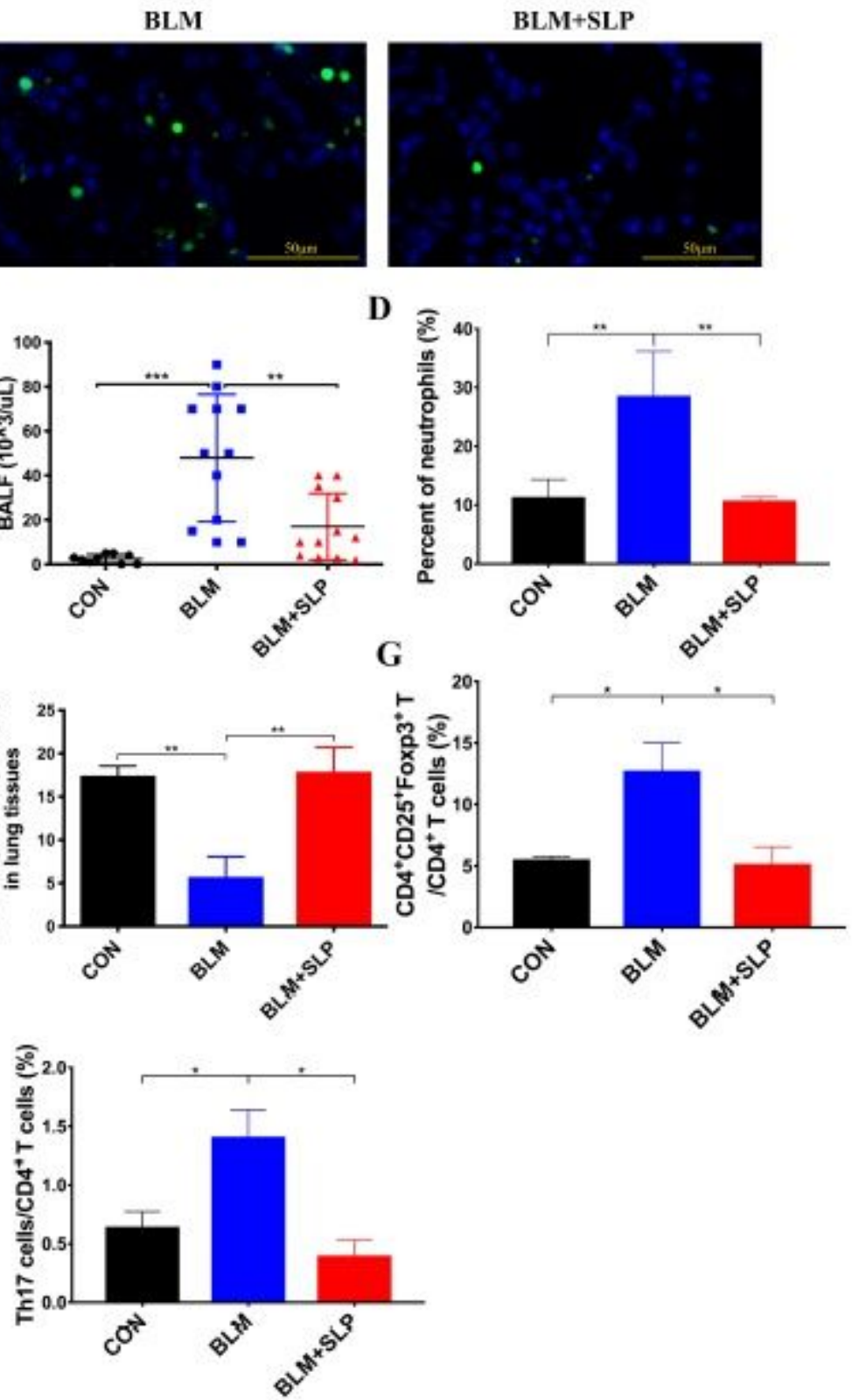

\section{Figure 2}

MSC SLP inhibited apoptosis and inflammatory cell infiltration induced by BLM. a TUNEL staining to detect apoptotic cells. b Total protein levels $\mathrm{c}$ total cell counts, and d neutrophil percentages in BALFs were assessed. e MPO activities in lung homogenates were measured. $f$ The percentages of CD4+ T cells, and g CD4+CD25+Foxp3+ Treg cells in the lungs were analyzed by flow cytometry. $\mathrm{h}$ The percentage of Th17 cells in the blood were analyzed by flow cytometry. $N=6-8$ in each group. The data shown are presented as the mean $\pm S D$, statistical differences were and assessed by performing one-way ANOVA. *P $<0.05 ; * \star P<0.01 ; * \star * P<0.001$ 
A
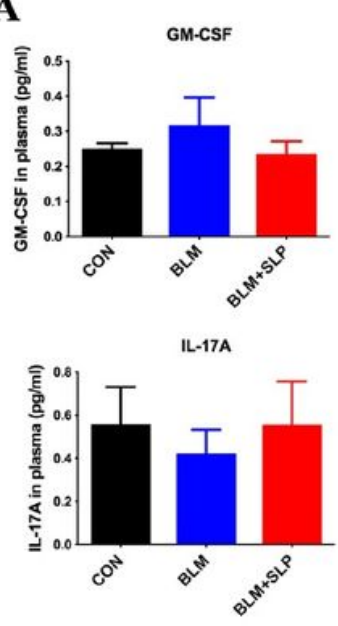

$11-4$

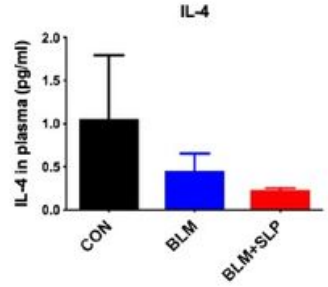

IFN-Y

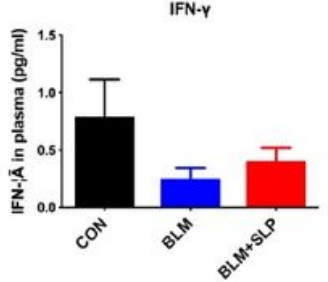

IL-17C
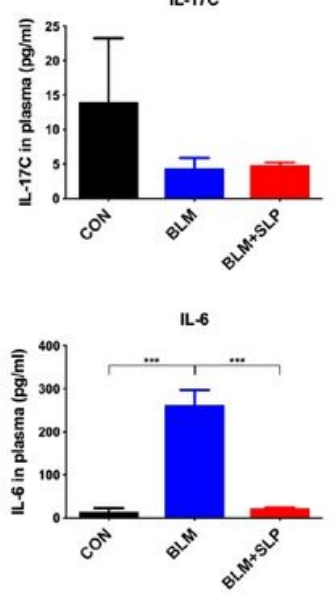
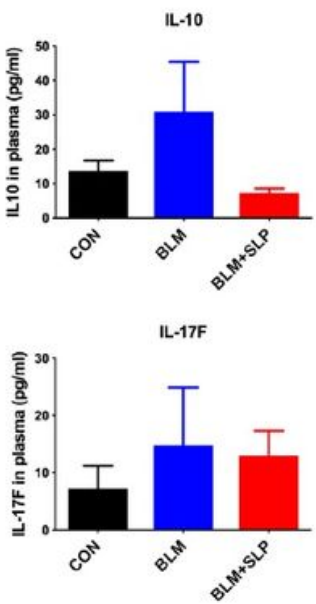

IL-23

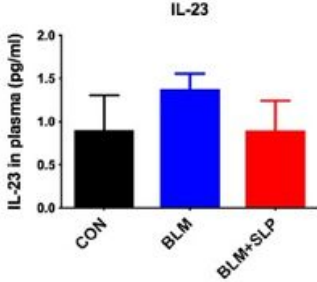

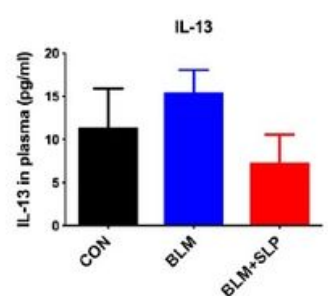

B

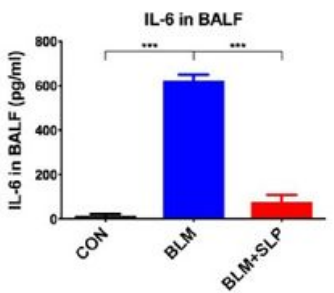

C

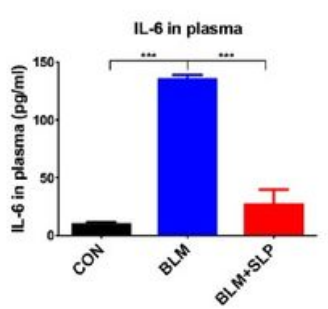

D
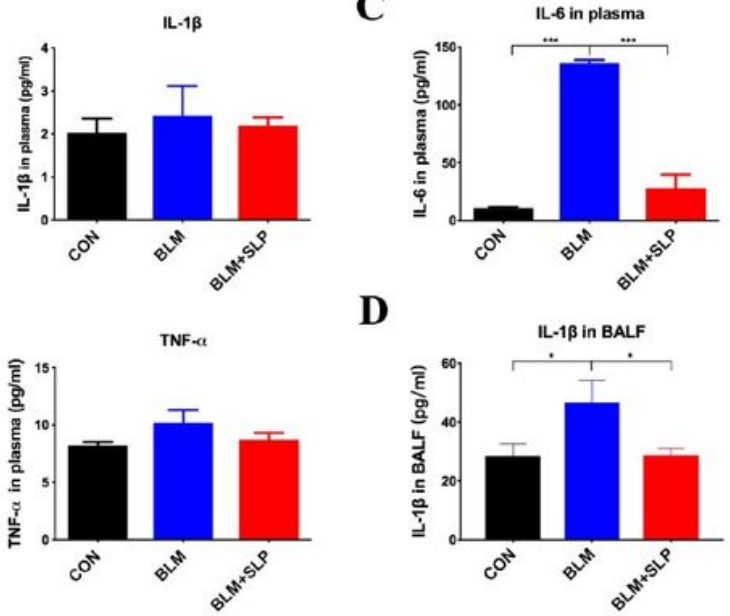

Figure 3

MSC SLP alleviated pro-inflammatory cytokine production induced by BLM. a MSD electrochemiluminescence assays. $b-c$ IL- 6 concentrations in BALF and plasma samples were measured by ELISA. $d$ IL-1 $\beta$ concentrations in BALFs were detected by ELISA. $N=6-8$ in each group. The data shown are presented as the mean $\pm S D$, and statistical differences were assessed by performing one-way ANOVA. ${ }^{*} \mathrm{P}<0.05 ; * \star * \mathrm{P}<0.001$ 
A

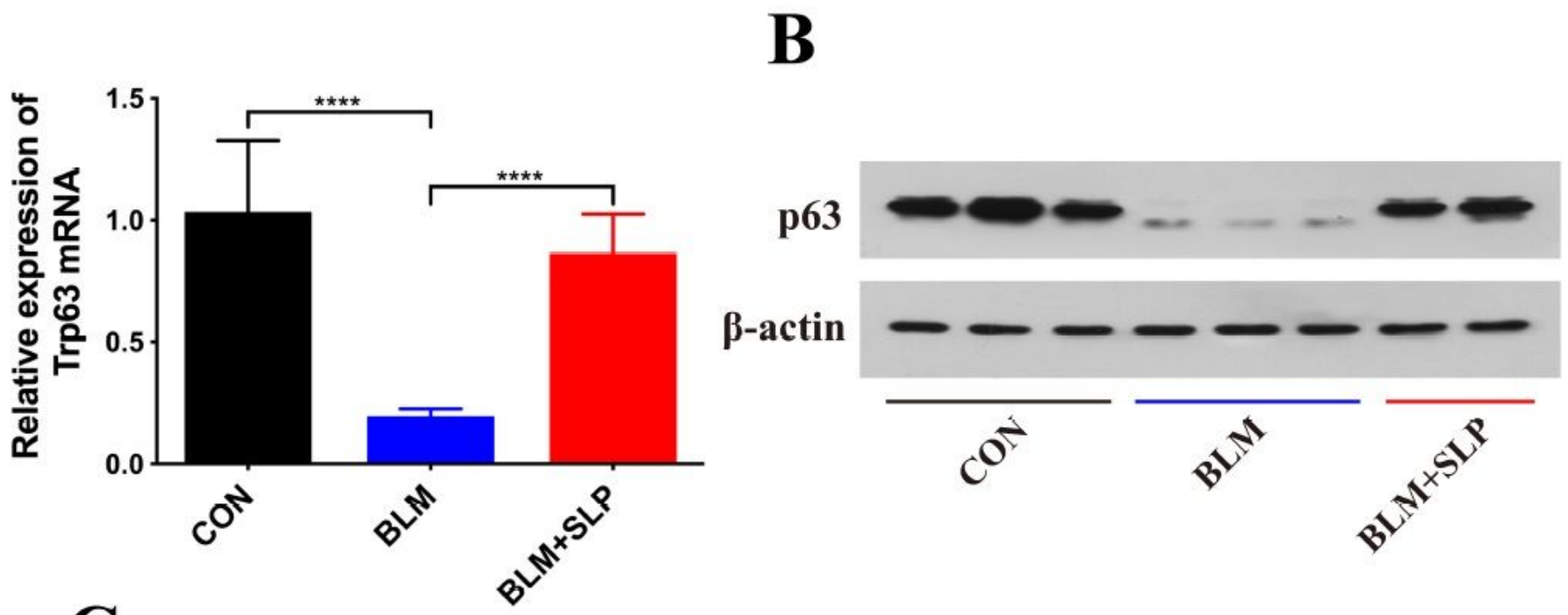

C

p63
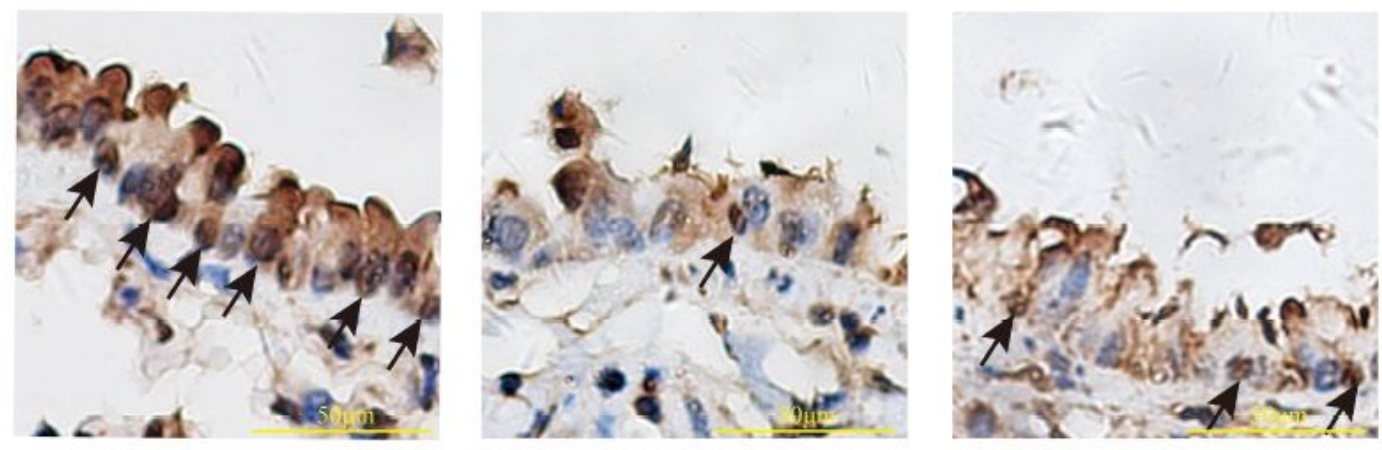

$c^{\hat{v}}$
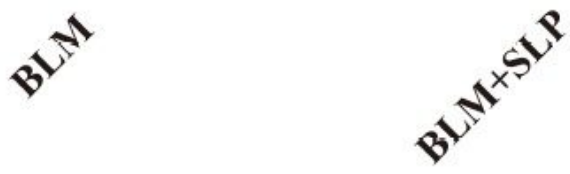

Figure 4

MSC SLP increased p63 expression. The expression of p63 in lung tissues was detected by a RT-qPCR, $b$ western blotting, and c IHC. Arrows indicated p63+ cells. $\mathrm{N}=6-8$ in each group. The data shown are presented as the mean $\pm S D$, and statistical differences were assessed by performing one-way ANOVA. $\star \star \star \star P<0.0001$ 


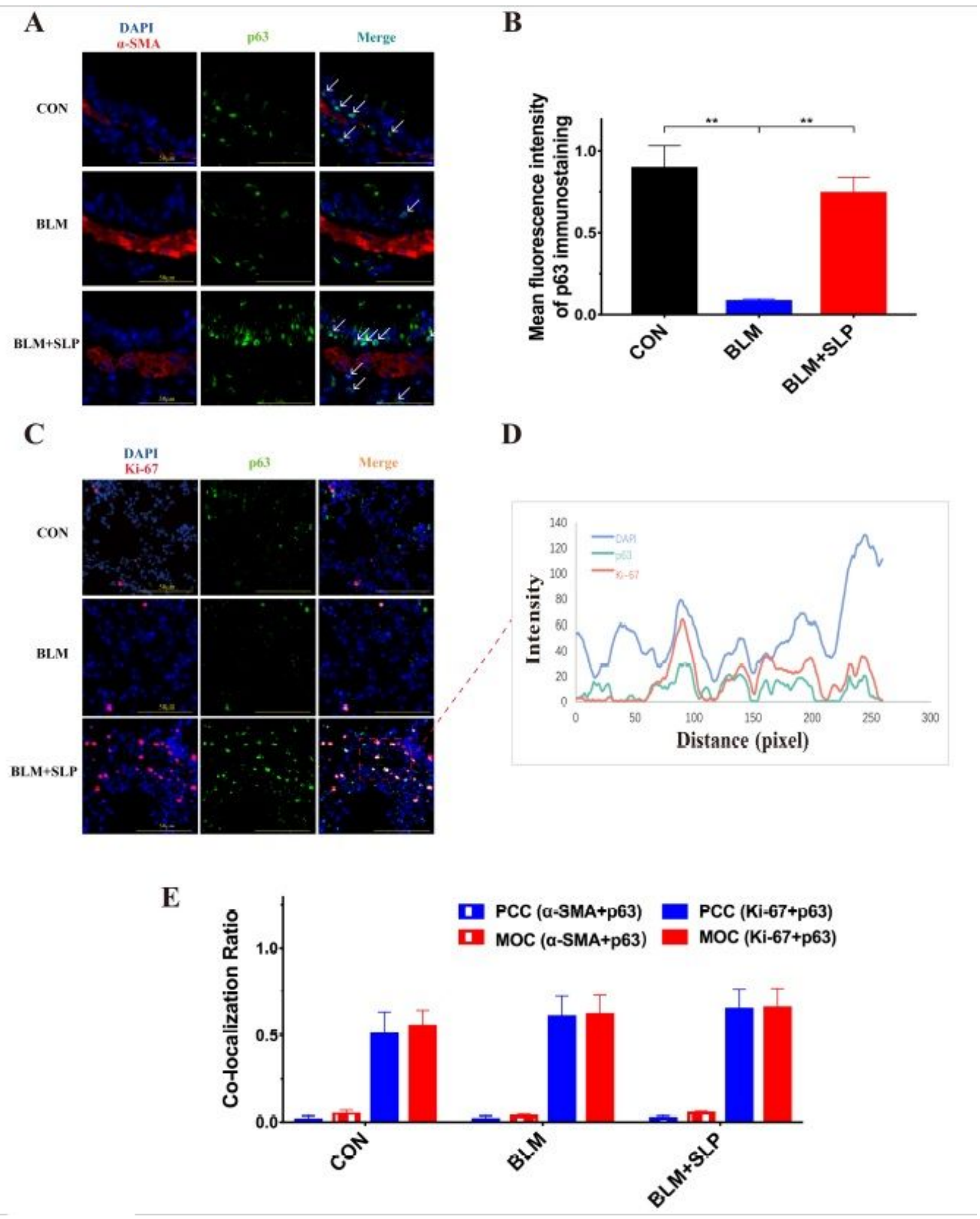

\section{Figure 5}

MSC promoted p63+ cell proliferation in the lungs. a Immunofluorescence staining with antibodies against p63 and a-SMA. b Quantitative analysis of p63 expression, as determined by immunofluorescence staining. c Immunofluorescence staining with antibodies against p63 and Ki-67. d Staining-intensity profiles showing signals from all three fluorescent channels. e PCC and MOC values. 
Ten fields were randomly selected for scoring. The data shown are presented as the mean \pm SD, and statistical differences were assessed by performing one-way ANOVA. ${ }^{*} \mathrm{P}<0.01$

A

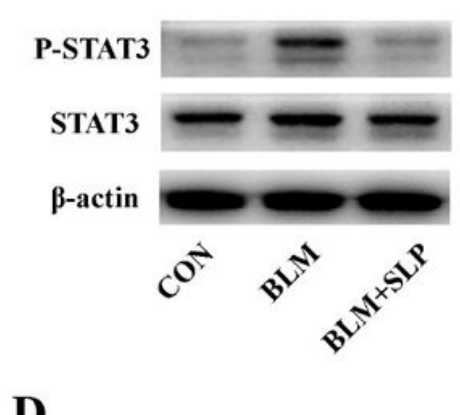

D

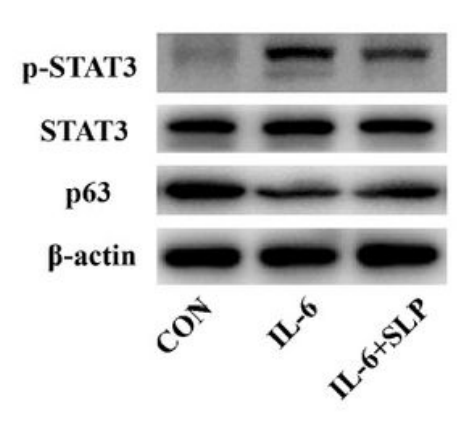

B

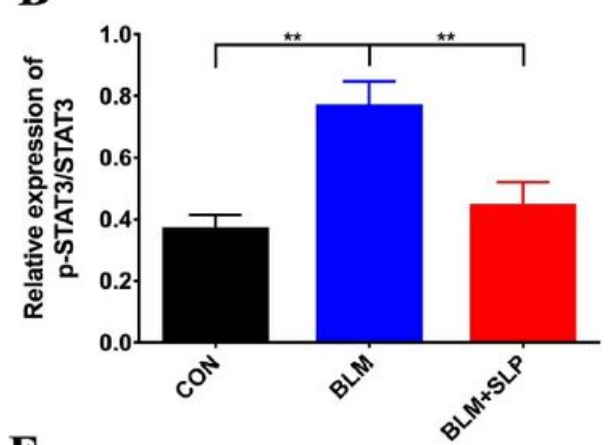

$\mathbf{E}$

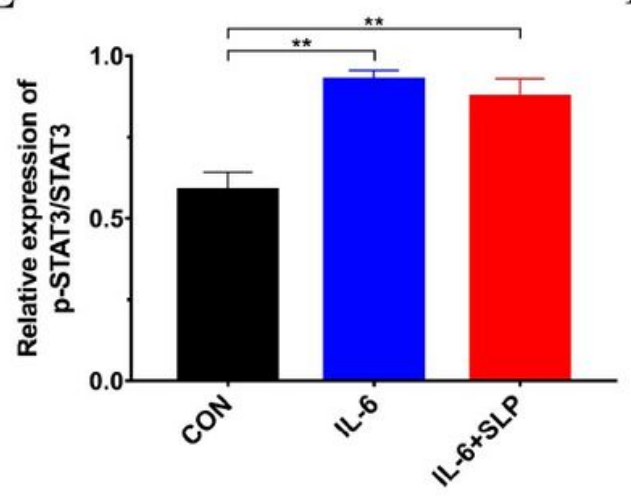

C

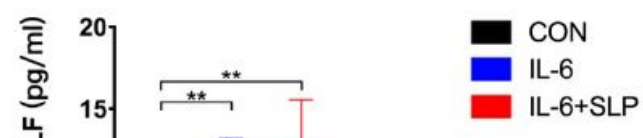

$\mathbf{F}$

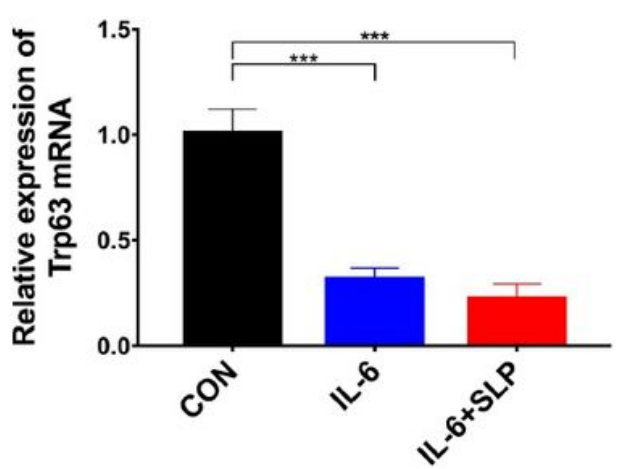

Figure 6

MSC SLP inhibited STAT3 phosphorylation, and rh IL-6 enhanced STAT3 phosphorylation to reduce p63 expression. a The levels of the p-STAT3, STAT3, and $\beta$-actin proteins were measured by western blotting. b Quantitative analysis of p-STAT3 expression. c The concentrations of $\mathrm{rh}$ IL- 6 in the lungs on days 1 and 2 after rh IL-6 administration. $d$ The protein levels of p-STAT3, STAT3, p63, and $\beta$-actin were measured by western blotting. e Quantitative analysis of p-STAT3 expression. f p63 mRNA-expression levels were measured by RT-qPCR. $N=6-8$ in each group. The data shown are presented as the mean $\pm S D$, and statistical differences were assessed by performing one-way ANOVA. $* * P \llbracket 0.01 ; * \star * P \llbracket 0.001$ 


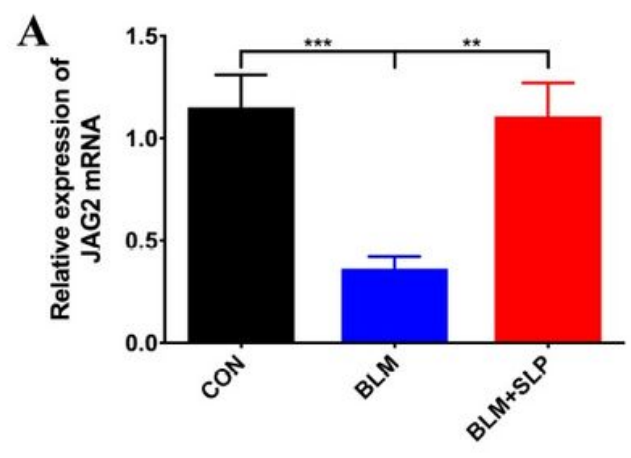

B

D

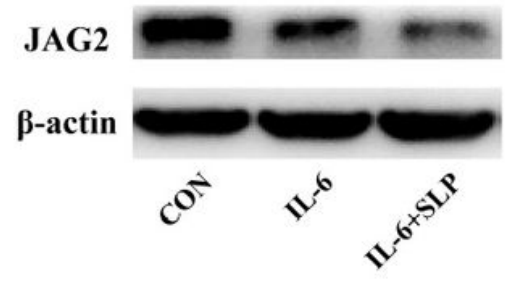

$\mathbf{E}$

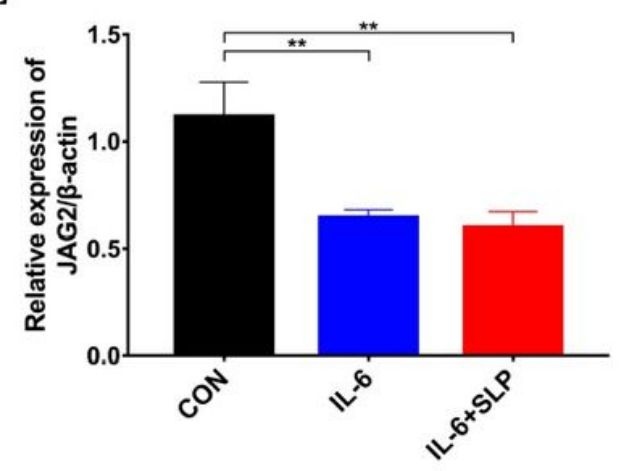

C

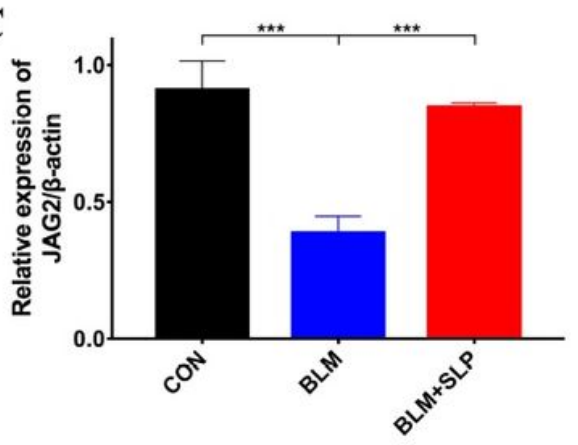

$\mathbf{F}$

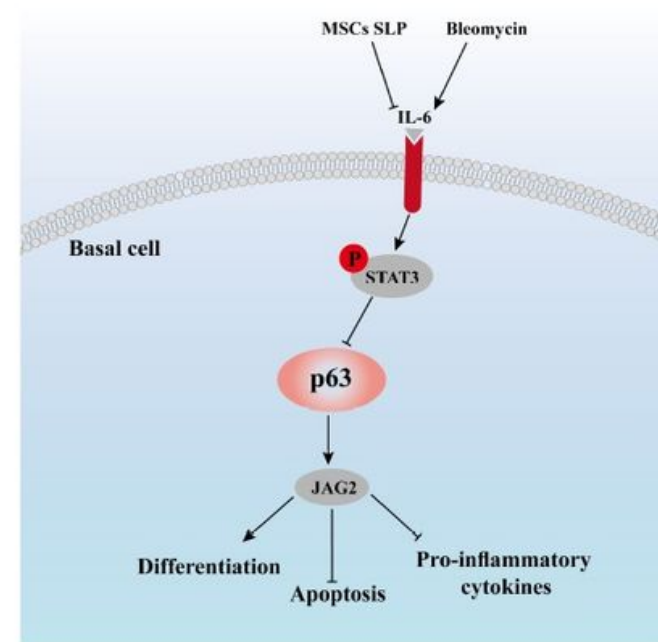

\section{Figure 7}

MSC SLP increased JAG2 expression by activating p63. a. JAG2 mRNA-expression levels in a mouse model of ALI. b The protein-expression levels of JAG2 and $\beta$-actin in a mouse model of ALI. c Quantitative analysis of JAG2 expression. $d$ The protein-expression levels of JAG2 and $\beta$-actin in mice treated with IL$6 \pm$ SLP. e Quantitative analysis of JAG2 expression. f Schematic model of how MSC SLP attenuates BLM-induced ALI. $N=6-8$ in each group. The data shown are presented as the mean $\pm S D$, and statistical differences were assessed by performing one-way ANOVA. ${ }^{\star * P}<0.01 ; \star \star \star P<0.001$ 
A

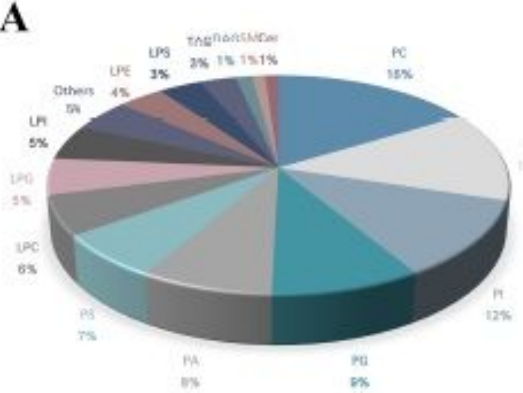

D

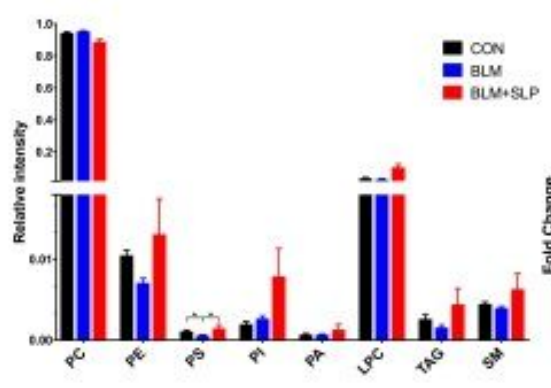

B

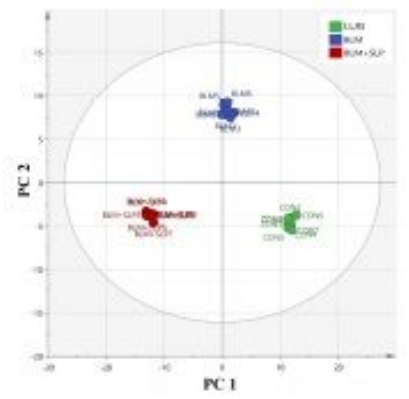

C

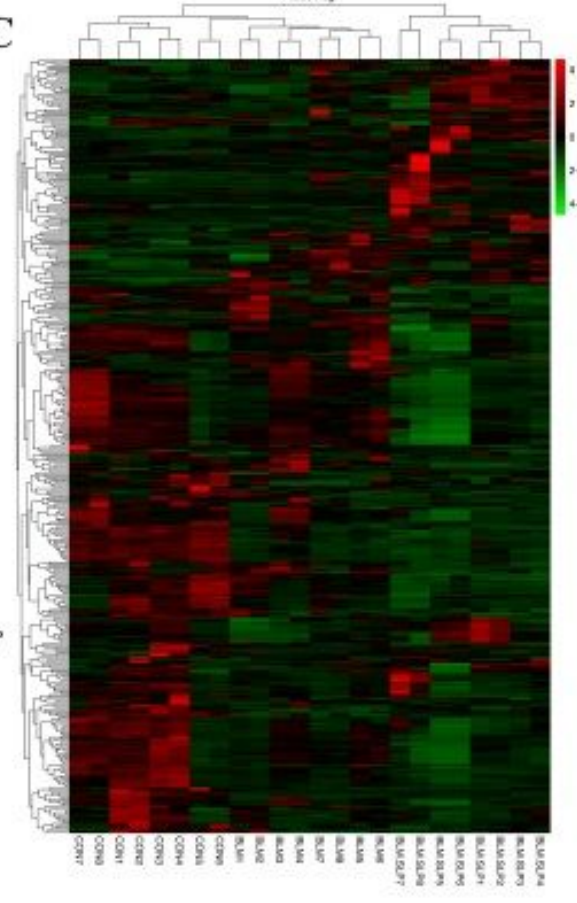

F

$\mathbf{E}$
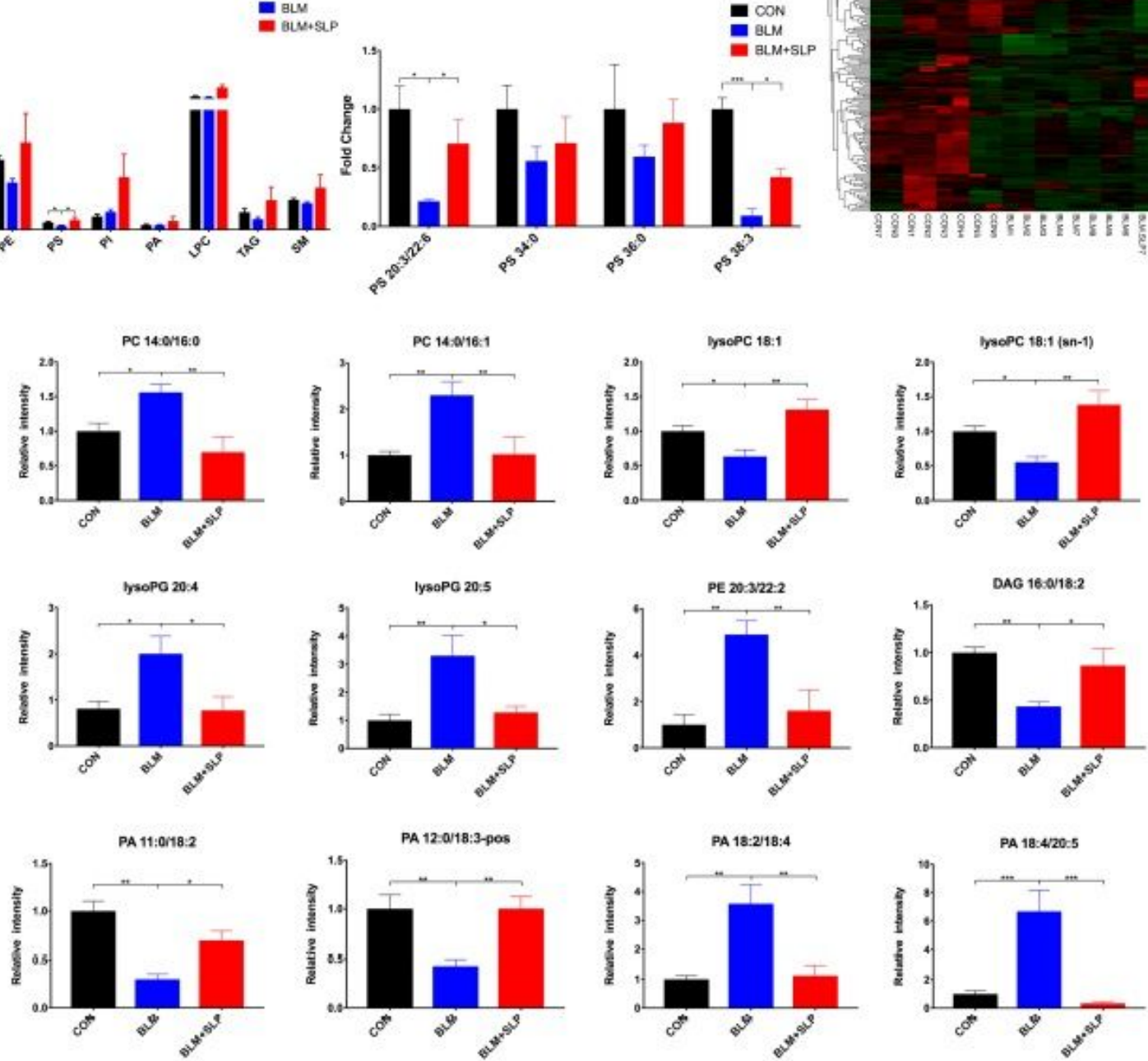

Figure 8

MSC SLP regulated the plasma lipid profile. a Lipid classes detected in the plasma. b Principal component analysis. c Clustering results depicted as a heatmap. d Relative MS-based intensity of major lipid species. e Normalized levels of individual PS lipid species. f Normalized levels of individual lipids in other classes. $\mathrm{N}=6-8$ in each group. The data shown are presented as the mean $\pm \mathrm{SD}$, and statistical differences were assessed by performing one-way ANOVA. ${ }^{*} \mathrm{P}<0.05 ; * \star \mathrm{P}<0.01 ; * \star * \mathrm{P}<0.001$

\section{Supplementary Files}


This is a list of supplementary files associated with this preprint. Click to download.

- Supplementary.pdf 